\title{
Efficacy and safety profile of boceprevir- or telaprevir-based triple therapy or dual peginterferon alfa-2a or alfa-2b plus ribavirin therapy in chronic hepatitis C: the real-world PegBase observational study
}

\author{
Alessandra Mangia a, Graham R. Fosterb, Christoph P. Berg', Manuela Curescu ${ }^{d}$, Victor De Ledinghen ${ }^{e}$, \\ François Habersetzerf, Spilios Manolakopoulos ${ }^{\mathfrak{g}}$, Elisa Negri ${ }^{h}$, George Papatheodoridisi, Silke Ahlers ${ }^{k}$, \\ Marco Castillo', Georgios Bakalos', Stefan Mauss ${ }^{m}$ on behalf of the PegBase Group Investigators
}

Abstract

Background The aim of the study was to determine the efficacy and safety of triple therapy with a first-generation protease inhibitor (PI; boceprevir, telaprevir) plus peginterferon alfa-2a or $-2 \mathrm{~b}$ plus ribavirin, and dual therapy (peginterferon alfa-2a or $-2 b$ plus ribavirin) in patients with chronic hepatitis $\mathrm{C}(\mathrm{CHC})$ in routine clinical practice.

Methods PegBase was an international, prospective, observational study in which 4441 patients with $\mathrm{CHC}$ were enrolled in 27 countries. This analysis focuses on results in 4100 treatment-naïve and previously treated patients treated with PI-based triple therapy or dual therapy, according to the discretion of the investigator and local standards of practice. The primary efficacy outcome was sustained virological response after 12-week follow up (SVR12).

Results SVR12 rates in treatment-naïve genotype (G) 1 patients were $56.6 \%$ and $62.9 \%$ for recipients of boceprevir plus peginterferon alfa-2a/ribavirin and boceprevir plus peginterferon alfa-2b/ribavirin, respectively, and $65.3 \%$ and $58.6 \%$ for recipients of telaprevir plus peginterferon alfa-2a/ribavirin and telaprevir plus peginterferon alfa- $\mathrm{b} /$ ribavirin, respectively. In previously treated patients assigned to these four regimens, SVR12 rates were $43.6 \%, 48.3 \%, 60.3 \%$ and $56.1 \%$, respectively. Among treatment-naïve patients assigned to peginterferon alfa-2a/ribavirin and peginterferon alfa-2b/ribavirin, respectively, SVR 12 rates were $49.2 \%$ and $41.9 \%$ in G1 patients, $75.7 \%$ and $83.3 \%$ in G2 patients, $65.9 \%$ and $65.9 \%$ in G3 patients, and $49.7 \%$, and $51.1 \%$ in G4 patients. The safety and tolerability of dual and triple therapy were consistent with previous reports.

Conclusion The efficacy and safety of first-generation PI-based triple-therapy and dual-therapy regimens in this real-world cohort were broadly comparable to those of previous studies.

Keywords Boceprevir, peginterferon, ribavirin, telaprevir, virological response

Ann Gastroenterol 2017; 30 (3): 1-17

\footnotetext{
${ }^{a}$ Liver Unit, IRCCS Hospital “Casa Sollievo della Sofferenza”, San Giovanni Rotondo, Italy; 'Institute of Cellular and Molecular Sciences, Queen Mary University of London, London, UK; ' Department of Internal Medicine, Medizinische Universitätsklinik Tübingen, Tübingen, Germany; ${ }^{\mathrm{d} C l i n i c}$ of Infectious Diseases, University of Medicine and Pharmacy Timișoara, Timișoara, Romania; ${ }^{~ H e p a t o l o g y ~ U n i t, ~ H o ̂ p i t a l ~ H a u t-L e ́ v e ̂ q u e, ~ C H U ~}$ Bordeaux, Pessac, France; 'Unité Hépatologie, Pôle Hépato-digestif, Hôpitaux Universitaires de Strasbourg, Inserm 1110, Université de Strasbourg, Strasbourg, France; ${ }^{g}$ Department of Gastroenterology, Polyclinic General Hospital, Athens, Greece; ${ }^{\mathrm{h}} \mathrm{UO}$ Malattie Infettive ed Epatologia, Azienda Ospedaliero - Universitaria di Parma, Parma, Italy; ${ }^{i}$ Athens University Medical School, Laiko General Hospital, Athens, Greece; ${ }^{k}$ PROMETRIS GmbH, Mannheim, Germany; 'F. Hoffmann-La Roche Ltd., Basel, Switzerland; ' ${ }^{\mathrm{m} C e n t e r ~ f o r ~ H I V ~ a n d ~ H e p a t o g a s t r o e n t e r o l o g y, ~ D u ̈ s s e l d o r f, ~ G e r m a n y ~}$

Conflict of interest: Alessandra Mangia: Advisory board: BMS, MSD, Gilead; Research grants: Janssen, MSD; Speakers' bureau: Gilead, BMS, Janssen. Graham R. Foster: Consultancy and speaker fees for F. Hoffmann-La Roche, Gilead, AbbVie, Merck, GSK, Janssen. Christoph P. Berg: Advisory board: AbbVie, BMS, Gilead, Janssen, MSD, F. Hoffmann-La Roche; Speaker honorarium: AbbVie, BMS, Gilead, Janssen, MSD, Roche. Manuela Curescu: None. Victor De Ledinghen: Advisory board: AbbVie, BMS, Gilead, MSD, Janssen; Speaker honorarium: AbbVie, BMS, Gilead, Janssen, MSD. François Habersetzer: Personal fees from F. Hoffmann-La Roche Ltd and Merck; Travel grants from Merck and Janssen. Spilios Manolakopoulos: Advisor/Lecturer: Gilead, BMS, Janssen, Abbvie, MSD, Novartis, Bayer; Research grants: BMS, Gilead. Elisa Negri: None. George Papatheodoridis: Grant/research support from AbbVie, Boehringer Ingelheim, BMS, Gilead, Janssen, MSD, and Regulus; Scientific consultant and/or advisory boards and/or lecturer for AbbVie, BMS, Gilead, GlaxoSmithKline, Janssen, MSD, Novartis, F. Hoffmann-La Roche; Safety Management Board member for Gilead. Silke Ahlers: Employee of PROMETRIS GmbH, the clinical research organization providing data management and statistical services to Roche. Marco Castillo: Employee of F. Hoffmann-La Roche Ltd, Basel, Switzerland. Georgios Bakalos: Employee of F. Hoffmann-La Roche Ltd, Basel, Switzerland. Stefan Mauss: Advisory board: AbbVie, BMS, Gilead, MSD; Speaker honorarium: AbbVie, BMS, Gilead, Janssen, MSD
} 


\section{Introduction}

Hepatitis $\mathrm{C}$ virus (HCV) is the most common infectious cause of chronic liver disease. More than 185 million people worldwide are infected with HCV, of whom 350,000 die each year [1]. The highest prevalence of chronic hepatitis $C$ (CHC) is in Asia, the Middle East and North Africa. Untreated $\mathrm{CHC}$ can result in cirrhosis, liver failure and hepatocellular carcinoma [1]. HCV genotype $(G) 1$ is the most prevalent among HCV genotypes, comprising almost half of all HCV infections [2]. Patients with $\mathrm{CHC}$ have a $15-30 \%$ risk of cirrhosis within 20 years [3], while the risk of hepatocellular carcinoma for people with cirrhosis is approximately $2-4 \%$ per year [4]. It has been estimated that HCV infection accounts for the loss of over 12,000 disability-adjusted life years worldwide [5].

The primary objective of $\mathrm{CHC}$ treatment is eradication of the virus from the host, usually characterized as a sustained virological response (SVR), defined by the absence of detectable HCV RNA in the serum 3-6 months after the end of treatment (EoT) [1]. Until 2011, the combination of peginterferon alfa and ribavirin for 24 or 48 weeks (dual therapy) was the standard of care for patients with $\mathrm{CHC}$, and produced an overall SVR rate of approximately $40 \%$. SVR rates with interferon-based therapy vary according to host and viral factors, such as HCV genotype, HCV RNA level, host IL28B genotype and the extent of hepatic fibrosis. Since 2011, direct-acting antivirals (DAAs) have been available for the treatment of $\mathrm{CHC}$. The first DAAs, boceprevir and telaprevir, were inhibitors (PI) of the HCV protease NS $3 / 4 \mathrm{~A}$ and, when added to peginterferon alfa/ribavirin as triple therapy, improved SVR rates to approximately $60-70 \%$ in G1 patients [6,7]. First-generation PI-based triple therapy is associated with a higher rate of hematological adverse events (AEs), in particular anemia, and potentially severe hypersensitivity reactions are common with telaprevir [6-9]. While tolerability was an issue, and the costs of treatment made DAAs unavailable for many patients, triple therapy became the standard of care for patients with G1 infection. Boceprevir and telaprevir have since been superseded by next-generation DAAs with improved efficacy and tolerability, and with the availability of interferon-free combinations of DAAs with higher efficacy and broader genotype coverage, these two drugs have been withdrawn from the US market [10-12]. However, availability and cost considerations mean that many patients still do not have access to newer DAAs, so that peginterferonbased regimens may still be relevant in some countries $[11,12]$.

The PegBase study is an international, prospective, observational study that was initiated after the first PIs became

Funding: F. Hoffmann-La Roche Ltd., Basel, Switzerland

Correspondence to: Alessandra Mangia, Liver Unit, IRCCS Hospital "Casa Sollievo della Sofferenza", San Giovanni Rotondo, Italy, Tel.: +390882 416375, Fax: +390882 416271, e-mail: a.mangia@tin.it

Received 30 September 2016; accepted 19 February 2017; published online 23 March 2017

DOI: https://doi.org/10.20524/aog.2017.0136 available in 2011 with the objective of characterizing the efficacy and safety of boceprevir- and telaprevir-based triple therapy, as well as dual therapy, in patients with $\mathrm{CHC}$ in routine clinical practice.

\section{Patients and methods}

The PegBase study was a prospective, international cohort study in patients with $\mathrm{CHC}$ conducted in 27 countries (Belgium, Egypt, Estonia, France, Germany, Greece, Hungary, Ireland, Italy, Kuwait, Lebanon, Former Yugoslav Republic of Macedonia, Morocco, Oman, Pakistan, Portugal, Qatar, Romania, Saudi Arabia, Serbia, Sweden, Switzerland, Syrian Arab Republic, Taiwan, Turkey, United Arab Emirates and the United Kingdom).

Adult patients with untreated or previously treated $\mathrm{CHC}$ were eligible if they had quantifiable HCV RNA at the start of treatment and were prescribed, as part of standard care according to local labeling, either dual combination therapy with peginterferon alfa-2a or $-2 b$ plus ribavirin, or boceprevir- or telaprevir-based triple therapy incorporating peginterferon alfa $2 \mathrm{a}$ or $2 \mathrm{~b}$ plus ribavirin. Patients with hepatitis B virus coinfection were excluded. Drug dosages and treatment durations were left to the discretion of the investigator and were to be determined according to the local label and standards of practice. Patients were followed up for 24 weeks after completion of treatment.

The current analysis includes results from HCV monoinfected patients who received dual peginterferon alfa/ribavirin therapy, and patients with HCV G1 infection who received boceprevir- or telaprevir-based triple therapy, and comprised the core population. A comprehensive list of exclusion criteria used to define the core population for this analysis is shown in Supplemental Table 1. The protocol was approved by the Independent Ethics Committee or Institutional Review Board at each center, and each patient provided written informed consent. The trial is registered with clinicaltrials.gov: NCT01447446.

\section{Study endpoints}

Virological response (VR) was defined as HCV RNA $<50 \mathrm{IU} / \mathrm{mL}$ (dual therapy) or undetectable HCV RNA (triple therapy), using a test with a lower limit of detection $\leq 50 \mathrm{IU} / \mathrm{mL}$. The primary endpoint of the study was SVR. When the trial was designed, SVR was defined as VR at 24 weeks post-treatment (SVR24); however, after the study was initiated, determination of SVR at 12 weeks post-treatment (SVR12) became an accepted definition for treatment success [13]. Both SVR12 and SVR24 were obtained and are presented. However, in this real-world study, patients who achieved an SVR12 may have been less likely to return for an assessment at 24-week follow up. SVR12 and SVR24 were defined as achievement of a VR $\geq 70$ and $\geq 140$ days after the day of last treatment. Scheduling of HCV 
RNA assessments was at the discretion of the local investigator. HCV RNA was measured using polymerase chain reaction according to standard methods. Secondary endpoints included on-treatment VR rates, relapse rates (defined as detectable HCV RNA during follow up in recipients of triple therapy or HCV RNA $\geq 50 \mathrm{IU} / \mathrm{mL}$ during follow up in recipients of dual therapy in patients with an EoT VR) and rates of breakthrough (HCV RNA detectable/ $\geq 50 \mathrm{IU} / \mathrm{mL}$ while still on treatment after an initial response), or rebound ( $\geq 1-\log _{10}$ increase in HCV RNA over nadir during treatment). Extended rapid VR (eRVR) was defined as a VR at weeks 4 and 12 of treatment in patients treated with telaprevir and a VR at weeks 8 and 24 in patients treated with boceprevir.

$\mathrm{VR}$ rates were also presented by fibrosis status. Investigators reported the method of assessment (biopsy, noninvasive or best guess) and the result of assessment (cirrhosis, transition to cirrhosis, no cirrhosis) on the electronic case report form (eCRF). For patients with a biopsy result, transition to cirrhosis was prespecified in the eCRF as ISHAK stage 5 or 4 (with nodules or $>3$ bridges), METAVIR stage 3 , Batts and Ludwig stage 3, Knodell stage 3 and Scheuer stage 3. Cirrhosis was defined as ISHAK stage 6 , METAVIR stage 4 , Batts and Ludwig stage 4 , Knodell stage 4 and Scheuer stage 4 . In the present analysis, cirrhosis and transition to cirrhosis were combined into one category. For patients with no documented biopsy result, the determination was based either on a noninvasive assessment (if documented in the patient record) or a "best guess" assessment. In such cases, one of the three categories (cirrhosis, transition to cirrhosis and no cirrhosis) was reported in the eCRF, based on the investigator's judgment without prespecified definitions.

Safety endpoints included AEs, serious AEs (SAEs), laboratory abnormalities and dose modifications of peginterferon alfa and ribavirin. AEs were recorded continuously. Laboratory (hematological) parameters were measured at the discretion of the investigator according to local guidelines.

\section{Statistical analysis}

For statistical analysis, no hypothesis testing was performed and the endpoints were analyzed by descriptive and exploratory methods. The planned sample size of 4000 patients included in the overall study was based on feasibility considerations and no formal sample size calculations were performed. All analyses reported here were conducted in the core study population.

VR rates were calculated with $95 \%$ confidence intervals, based on the normal approximation to the binomial distribution. Subgroup analyses were performed by HCV genotype (dual therapy only), presence or absence of transition to cirrhosis or cirrhosis, host rs12979860 IL28B gene polymorphism, and baseline HCV RNA level ( $\leq 400,000$ or $>400,000 \mathrm{IU} / \mathrm{mL})$. For patients with missing virological data during the treatment period, if the HCV measurements before and after the missing measurement both showed VR, the missing measurement was assumed to also show VR. Similarly, for patients with a missing EoT measurement, if the next available measurement post-treatment showed VR, VR was assumed for EoT. In all other cases, missing measurements were considered as nonresponse.

AEs were categorized according to the Medical Dictionary for Drug Regulatory Affairs version 18.1 and expressed as the proportion of patients with an AE. Laboratory safety variables were converted to SI units if required, with the results transformed to a standard reference range to allow comparison of results from different laboratories.

\section{Results}

A total of 4441 patients were enrolled and followed up between 1 September 2011 and 24 July 2015. Of these, 4352 individuals received at least one dose of study medication and were included in the safety population. A total of 252 patients in the safety population were excluded from the core population (Supplemental Table 2). The core population for analysis comprised 4100 patients, of whom 1292 were assigned to boceprevir- or telaprevir-based triple therapy (all infected with HCV G1). Baseline characteristics are shown by treatment regimen and treatment history for patients receiving triple therapy in Table 1 and for treatment-naïve patients receiving dual therapy in Table 2 .

Among patients assigned to triple-therapy, $70.2 \%$ received telaprevir and $29.8 \%$ boceprevir (Fig. 1). Patient disposition is shown in Table 3 and reasons for premature withdrawal from treatment are shown in Table 4.

\section{VR in treatment-naïve patients receiving triple therapy}

Among treatment-naïve patients assigned to triple therapy, SVR12 rates were $56.6 \%$ and $62.9 \%$ for patients who received boceprevir in combination with peginterferon alfa-2a/ribavirin and peginterferon alfa-2b/ribavirin, respectively, and $65.3 \%$ and $58.6 \%$ for patients who received telaprevir in combination with peginterferon alfa-2a/ribavirin and peginterferon alfa- $2 \mathrm{~b} /$ ribavirin, respectively (Fig. 2A). Corresponding relapse rates in patients evaluable for relapse in these four treatment groups were $14.1 \%, 18.5 \%, 15.7 \%$ and $5.6 \%$, respectively (Fig. 2B), whilst breakthrough or rebound occurred in $8.2 \%, 14.3 \%, 8.1 \%$ and $23.1 \%$, respectively, of patients evaluable for breakthrough or rebound (Table 5).

An eRVR was achieved by $46.9 \%$ and $42.9 \%$ of patients receiving boceprevir with peginterferon alfa-2a/ribavirin and peginterferon alfa- $2 \mathrm{~b} /$ ribavirin, respectively, and $42.8 \%$ and $37.9 \%$ of patients receiving telaprevir with peginterferon alfa-2a/ribavirin and peginterferon alfa-2b/ribavirin, respectively.

SVR12 rates were consistently higher in non-cirrhotic than in cirrhotic patients and in patients with IL28B CC genotypes than in those with non-CC genotypes across the four treatment groups (Table 6). SVR12 rates also tended to be higher among 
Table 1 Baseline patient and disease characteristics - triple therapy

\begin{tabular}{|c|c|c|c|c|}
\hline Characteristic & $\begin{array}{c}\text { Boceprevir plus } \\
\text { PegIFN alfa-2a/RBV }\end{array}$ & $\begin{array}{l}\text { Boceprevir plus } \\
\text { PegIFN alfa-2b/RBV }\end{array}$ & $\begin{array}{c}\text { Telaprevir } \\
\text { PegIFN alfa-2a/RBV }\end{array}$ & $\begin{array}{c}\text { Telaprevir } \\
\text { PegIFN alfa-2b/RBV }\end{array}$ \\
\hline Treatment-naïve & $\mathrm{N}=143$ & $\mathrm{~N}=35$ & $\mathrm{~N}=\mathbf{2 8 5}$ & $\mathrm{N}=\mathbf{2 9}$ \\
\hline Female, n (\%) & $49(34)$ & $12(34)$ & $118(41)$ & $8(28)$ \\
\hline \multicolumn{5}{|l|}{ Race, n (\%) } \\
\hline Caucasian/white & $130(91)$ & $33(94)$ & $256(90)$ & $29(100)$ \\
\hline Black & $5(3)$ & $1(3)$ & $11(4)$ & - \\
\hline Asian/Oriental & $8(6)$ & $1(3)$ & $17(6)$ & - \\
\hline Other & - & - & $1(<1)$ & - \\
\hline Mean age, years (SD) & $47.8(11.0)$ & $48.8(12.5)$ & $49.4(11.7)$ & $52.8(9.7)$ \\
\hline Mean body mass index, $\mathrm{kg} / \mathrm{m}^{2}(\mathrm{SD})$ & $26.3(4.9)$ & $25.6(4.9)$ & $25.9(4.2)$ & $27.2(4.9)$ \\
\hline Mean HCV RNA, $\log _{10}$ IU/mL (SD) & $6.1(0.7)$ & $6.0(0.8)$ & $6.0(0.7)$ & $5.8(0.7)$ \\
\hline HCV RNA >400,000 IU/mL, n (\%) & $111(78)$ & $27(79)$ & $212(74)$ & $20(69)$ \\
\hline \multicolumn{5}{|l|}{ Method of assessing liver fibrosis, $n$ (\%) } \\
\hline Biopsy & $49(34)$ & $18(51)$ & $87(31)$ & $6(21)$ \\
\hline Noninvasive & $83(58)$ & $15(43)$ & $174(61)$ & $21(72)$ \\
\hline Not assessed or best guess & $11(8)$ & $2(6)$ & $24(8)$ & $2(7)$ \\
\hline \multicolumn{5}{|l|}{ Liver fibrosis status, n (\%) } \\
\hline Cirrhosis & $32(22)$ & $8(23)$ & $61(21)$ & $12(41)$ \\
\hline Transition to cirrhosis & $23(16)$ & $4(11)$ & $37(13)$ & $6(21)$ \\
\hline No cirrhosis & $88(62)$ & $23(66)$ & $187(66)$ & $11(38)$ \\
\hline \multicolumn{5}{|l|}{ IL28B rs12979860 host genotype, n (\%) } \\
\hline $\mathrm{CC}$ & $25 / 80(31)$ & $5 / 15(33)$ & $29 / 143(20)$ & $3 / 19(16)$ \\
\hline TC & $39 / 80(49)$ & $8 / 15(53)$ & $82 / 143(57)$ & $12 / 19(63)$ \\
\hline $\mathrm{TT}$ & $16 / 80(20)$ & $2 / 15(13)$ & $32 / 143(22)$ & $4 / 19(21)$ \\
\hline Median duration of infection, years & 20.0 & 23.0 & 18.0 & 12.5 \\
\hline Patients with type 2 diabetes, n (\%) & $11(7.7)$ & $2(5.7)$ & $20(7.0)$ & $1(3.4)$ \\
\hline Previously treated & $\mathrm{N}=149$ & $\mathrm{~N}=58$ & $\mathrm{~N}=536$ & $\mathrm{~N}=57$ \\
\hline Female, n (\%) & $59(40)$ & $25(43)$ & $213(40)$ & $35(61)$ \\
\hline \multicolumn{5}{|l|}{ Race, n (\%) } \\
\hline Caucasian/white & $143(96)$ & $55(95)$ & $515(96)$ & $57(100)$ \\
\hline Black & $2(1)$ & - & $7(1)$ & - \\
\hline Asian/Oriental & $3(2)$ & $3(5)$ & $13(2)$ & - \\
\hline Other & $1(<1)$ & - & $1(<1)$ & - \\
\hline Mean age, years (SD) & $54.3(8.1)$ & $56.9(9.6)$ & $54.0(9.5)$ & $57.1(9.3)$ \\
\hline Mean body mass index, $\mathrm{kg} / \mathrm{m}^{2}$ (SD) & $27.2(5.0)$ & $26.0(4.0)$ & $26.7(4.5)$ & $28.6(6.4)$ \\
\hline Mean HCV RNA, $\log _{10}$ IU/mL (SD) & $6.1(0.7)$ & $6.1(0.6)$ & $6.1(0.7)$ & $5.8(0.8)$ \\
\hline HCV RNA >400,000 IU/mL, n (\%) & $113(76)$ & $44(76)$ & $417(78)$ & $41(72)$ \\
\hline \multicolumn{5}{|l|}{ Method of assessing liver fibrosis, n (\%) } \\
\hline Biopsy & $35(23)$ & $7(12)$ & $66(12)$ & $8(14)$ \\
\hline Noninvasive & $91(61)$ & $42(72)$ & $367(68)$ & $42(74)$ \\
\hline Not assessed or best guess & $23(15)$ & $9(16)$ & $103(19)$ & $7(12)$ \\
\hline
\end{tabular}


Table 1 (Continued)

\begin{tabular}{|c|c|c|c|c|}
\hline Characteristic & $\begin{array}{c}\text { Boceprevir plus } \\
\text { PegIFN alfa-2a/RBV }\end{array}$ & $\begin{array}{c}\text { Boceprevir plus } \\
\text { PegIFN alfa-2b/RBV }\end{array}$ & $\begin{array}{c}\text { Telaprevir } \\
\text { PegIFN alfa-2a/RBV }\end{array}$ & $\begin{array}{c}\text { Telaprevir } \\
\text { PegIFN alfa-2b/RBV }\end{array}$ \\
\hline Previously treated & $\mathrm{N}=149$ & $\mathrm{~N}=58$ & $\mathrm{~N}=536$ & $\mathrm{~N}=57$ \\
\hline \multicolumn{5}{|l|}{ Liver fibrosis status, $\mathrm{n}(\%)$} \\
\hline Cirrhosis & $56(38)$ & $18(31)$ & $156(29)$ & $14(25)$ \\
\hline Transition to cirrhosis & $27(18)$ & $11(19)$ & $87(16)$ & $11(19)$ \\
\hline No cirrhosis & $66(44)$ & $29(50)$ & $293(55)$ & $32(56)$ \\
\hline \multicolumn{5}{|l|}{$\begin{array}{l}\text { IL28B rs12979860 host genotype, } \mathrm{n} / \mathrm{N} \\
\text { (\%) }\end{array}$} \\
\hline $\mathrm{CC}$ & $10 / 82(12)$ & $5 / 37(14)$ & $43 / 288(15)$ & $5 / 42(12)$ \\
\hline $\mathrm{TC}$ & $51 / 82(62)$ & $25 / 37(68)$ & $179 / 288(62)$ & $27 / 42(64)$ \\
\hline $\mathrm{TT}$ & $21 / 82(26)$ & $7 / 37(19)$ & $65 / 288(23)$ & $10 / 42(24)$ \\
\hline Negative & - & - & $1 / 288(<1)$ & - \\
\hline Median duration of infection, years & 21.0 & 17.0 & 21.0 & 8.0 \\
\hline Patients with type 2 diabetes, n (\%) & $24(16.1)$ & $10(17.2)$ & $68(12.7)$ & $1(1.8$ \\
\hline \multicolumn{5}{|l|}{ Response to prior treatment, $\mathrm{n}(\%)$} \\
\hline Relapse & $44(30)$ & $19(33)$ & $177(33)$ & $27(48)$ \\
\hline Breakthrough & $5(3)$ & $3(5)$ & $37(7)$ & $2(4)$ \\
\hline Non-response & $82(55)$ & $28(48)$ & $248(47)$ & $17(30)$ \\
\hline Other & $17(11)$ & $7(12)$ & $68(13)$ & $10(18)$ \\
\hline Missing & $1(1)$ & $1(2)$ & $6(1)$ & $1(2)$ \\
\hline
\end{tabular}

IL28B rs12979860 genotype was known for 257 treatment-naïve patients and 449 treatment-experienced patients; percentages are calculated from those with available data. One treatment-experienced patient was negative for rs12979860

HCV, hepatitis C virus; PegIFN, peginterferon; RBV, ribavirin; SD, standard deviation

patients with low baseline HCV RNA levels $(\leq 400,000 \mathrm{IU} / \mathrm{mL})$, although this was not the case in the small number of patients with low HCV RNA levels assigned to boceprevir plus peginterferon alfa-2a/ribavirin $(n=32$; Table 6$)$.

\section{VR in previously treated patients receiving triple therapy}

SVR12 rates were generally lower in previously treated patients than in treatment-naive patients assigned to the same treatment group (Tables 5, 6). Among previously treated patients assigned to triple therapy, SVR12 rates were $43.6 \%$ and $48.3 \%$ for patients who received boceprevir plus peginterferon alfa-2a/ribavirin and peginterferon alfa- $2 \mathrm{~b} /$ ribavirin, respectively and $60.3 \%$ and $56.1 \%$ for patients who received telaprevir plus peginterferon alfa-2a/ribavirin and peginterferon alfa-2b/ribavirin, respectively (Fig. 2C). Corresponding relapse rates in these four treatment groups were $28.1 \%, 22.2 \%, 12.6 \%$ and $8.6 \%$, respectively, among patients evaluable for relapse (Fig. 2D), whilst breakthrough or rebound occurred in $9.2 \%, 17.0 \%, 18.7 \%$ and $20.8 \%$, respectively, of patients evaluable for breakthrough or rebound (Table 5).

Consistently with trends observed in treatment-naïve patients, SVR12 rates were generally higher among previously treated patients who were non-cirrhotic compared to those who were cirrhotic, and among those with low baseline HCV RNA levels (Table 6). SVR12 rates tended to be higher in patients with CC than in those with non-CC genotypes, although the number of patients with CC genotypes was very small among previously treated patients (i.e., 3 of 4 groups had $\leq 10$ patients) and the trend was not observed consistently in all treatment groups.

SVR12 rates were generally, but not exclusively, higher in patients with a prior relapse than among those with a prior breakthrough or non-response (Table 6). When all four triple therapy groups were combined, the overall response rates in patients with a prior relapse, breakthrough or non-response were $73.8 \%$ (197/267), 55.3\% (26/47) and 44.3\% (166/375), respectively.

\section{Safety in patients receiving triple therapy}

Across the four triple-therapy treatment groups, the overall incidence of AEs ranged from $76.7 \%$ to $90.7 \%$ and the overall incidence of SAEs ranged from $4.7 \%$ to $19.9 \%$ (Table 7 ). The spectrum and frequency of individual AEs was similar to that reported previously, with anemia being the most frequently reported AE. Safety-related dose modifications were required 
Table 2 Baseline patient and disease characteristics of patients assigned to dual therapy

\begin{tabular}{|c|c|c|}
\hline Characteristic & $\begin{array}{c}\text { PegIFN } \\
\text { alfa-2a/RBV }\end{array}$ & $\begin{array}{c}\text { PegIFN } \\
\text { alfa-2b/RBV }\end{array}$ \\
\hline Treatment-naïve & $\mathrm{N}=1964$ & $\mathrm{~N}=414$ \\
\hline Female, n (\%) & $773(39)$ & $210(51)$ \\
\hline \multicolumn{3}{|l|}{ Race, n (\%) } \\
\hline Caucasian/white & $1709(87)$ & $370(89)$ \\
\hline Black & $29(1)$ & $2(<1)$ \\
\hline Asian/Oriental & $222(11)$ & $42(10)$ \\
\hline Other & $4(<1)$ & - \\
\hline Mean age, years (SD) & $46.7(13.0)$ & $49.1(12.8)$ \\
\hline \multicolumn{3}{|l|}{ Genotype, n (\%) } \\
\hline 1 & $815(41.5)$ & $198(47.8)$ \\
\hline 2 & $268(13.6)$ & $78(18.8)$ \\
\hline 3 & $528(26.9)$ & $91(22.0)$ \\
\hline 4 & $340(17.3)$ & 45 (10.9) \\
\hline 5 & $5(0.3)$ & $1(0.2)$ \\
\hline 6 & $8(0.4)$ & $1(0.2)$ \\
\hline $\begin{array}{l}\text { Mean body mass index, } \\
\mathrm{kg} / \mathrm{m}^{2}(\mathrm{SD})\end{array}$ & $26.4(4.5)$ & $25.6(4.7)$ \\
\hline $\begin{array}{l}\text { Mean HCV RNA, } \log _{10} \\
\text { IU/mL (SD) }\end{array}$ & $5.8(0.9)$ & $5.8(0.9)$ \\
\hline $\begin{array}{l}\text { HCV RNA >400,000 } \\
\text { IU/mL, n (\%) }\end{array}$ & $1305(66.5)$ & $275(66.4)$ \\
\hline \multicolumn{3}{|l|}{$\begin{array}{l}\text { Method of assessing } \\
\text { liver fibrosis, } \mathrm{n} / \mathrm{N}(\%)\end{array}$} \\
\hline Biopsy & $442 / 1963(23)$ & $84 / 414(20)$ \\
\hline Noninvasive & $1152 / 1963(59)$ & $250 / 414(60)$ \\
\hline $\begin{array}{l}\text { Not assessed or best } \\
\text { guess }\end{array}$ & $369 / 1963$ (19) & $80 / 414(19)$ \\
\hline \multicolumn{3}{|l|}{$\begin{array}{l}\text { Liver fibrosis status, } \\
\mathrm{n} / \mathrm{N}(\%)\end{array}$} \\
\hline Cirrhosis & $234 / 1963$ (12) & $47 / 414(11)$ \\
\hline Transition to cirrhosis & 265/1963 (13) & $55 / 414(13)$ \\
\hline No cirrhosis & $1464 / 1963(75)$ & $312 / 414(75)$ \\
\hline \multicolumn{3}{|l|}{$\begin{array}{l}\text { IL28B rs12979860 host } \\
\text { genotype, } \mathrm{n} / \mathrm{N}(\%)^{\mathrm{a}}\end{array}$} \\
\hline $\mathrm{CC}$ & $468 / 1243(38)$ & $81 / 246(33)$ \\
\hline $\mathrm{TC}$ & $617 / 1243(50)$ & $126 / 246(51)$ \\
\hline TT & $157 / 1243(13)$ & $39 / 246(16)$ \\
\hline $\begin{array}{l}\text { Median duration of } \\
\text { infection, years }\end{array}$ & 12.0 & 13.0 \\
\hline $\begin{array}{l}\text { Patients with type } 2 \\
\text { diabetes, } \mathrm{n}(\%)\end{array}$ & $179(9.1)$ & $43(10.4)$ \\
\hline
\end{tabular}

$($ Contd...)
Table 2 (Continued)

\begin{tabular}{|c|c|c|}
\hline Treatment-naïve & $\begin{array}{l}\text { PegIFN alfa- } \\
\text { 2a/RBV }\end{array}$ & $\begin{array}{c}\text { PegIFN } \\
\text { alfa-2b/RBV }\end{array}$ \\
\hline Previously treated & $\mathrm{N}=348$ & $\mathrm{~N}=82$ \\
\hline Female, n (\%) & $137(39)$ & $40(49)$ \\
\hline \multicolumn{3}{|l|}{ Race, n (\%) } \\
\hline Caucasian/white & $313(90)$ & $73(89)$ \\
\hline Black & $4(1)$ & $2(2)$ \\
\hline Asian/Oriental & $30(9)$ & $7(9)$ \\
\hline Other & $1(<1)$ & - \\
\hline Mean age, years (SD) & $51.6(11.0)$ & $54.6(10.6)$ \\
\hline \multicolumn{3}{|l|}{ Genotype, n (\%) } \\
\hline 1 & $173(49.7)$ & $46(56.1)$ \\
\hline Non-1 & $175(50.3)$ & $36(43.9)$ \\
\hline $\begin{array}{l}\text { Mean body mass index, } \\
\mathrm{kg} / \mathrm{m}^{2}(\mathrm{SD})\end{array}$ & $26.5(4.6)$ & $27.0(4.5)$ \\
\hline $\begin{array}{l}\text { Mean HCV RNA, } \\
\log _{10} \mathrm{IU} / \mathrm{mL} \text { (SD) }\end{array}$ & $5.9(0.8)$ & $5.9(0.7)$ \\
\hline $\begin{array}{l}\text { HCV RNA >400,000 } \\
\mathrm{IU} / \mathrm{mL}, \mathrm{n}(\%)\end{array}$ & $251(72.1)$ & $60(73.2)$ \\
\hline \multicolumn{3}{|l|}{$\begin{array}{l}\text { Method of assessing } \\
\text { liver fibrosis, } \mathrm{n}(\%)\end{array}$} \\
\hline Biopsy & $34(10)$ & $6(7)$ \\
\hline Noninvasive & $221(64)$ & $60(73)$ \\
\hline $\begin{array}{l}\text { Not assessed or best } \\
\text { guess }\end{array}$ & $93(27)$ & $16(20)$ \\
\hline \multicolumn{3}{|l|}{ Liver fibrosis status, n (\%) } \\
\hline Cirrhosis & $76(22)$ & $15(18)$ \\
\hline Transition to cirrhosis & $64(18)$ & $15(18)$ \\
\hline No cirrhosis & $208(60)$ & $52(63)$ \\
\hline \multicolumn{3}{|l|}{$\begin{array}{l}\text { IL28B rs12979860 host } \\
\text { genotype, } \mathrm{n}(\%)^{*}\end{array}$} \\
\hline $\mathrm{CC}$ & $69 / 219(32)$ & $16 / 51(31)$ \\
\hline TC & $117 / 219(53)$ & $25 / 51(49)$ \\
\hline TT & $33 / 219(15)$ & $10 / 51(20)$ \\
\hline $\begin{array}{l}\text { Median duration of } \\
\text { infection, years }\end{array}$ & 17.0 & 15.0 \\
\hline $\begin{array}{l}\text { Patients with type } 2 \\
\text { diabetes, } \mathrm{n}(\%)\end{array}$ & $41(11.8)$ & $13(15.9)$ \\
\hline
\end{tabular}

${ }^{*}$ One patient assigned to peginterferon alfa-2a plus ribavirin was negative for rs 12979860

HCV, hepatitis C virus; PegIFN, peginterferon; RBV, ribavirin; SD, standard deviation

more frequently for ribavirin (range $36.0 \%$ to $46.4 \%$ ) than for peginterferon alfa (range $10.5 \%$ to $19.4 \%$ ) across the four treatment groups. Anemia was the most frequently cited reason for ribavirin dose modifications, whilst neutropenia 


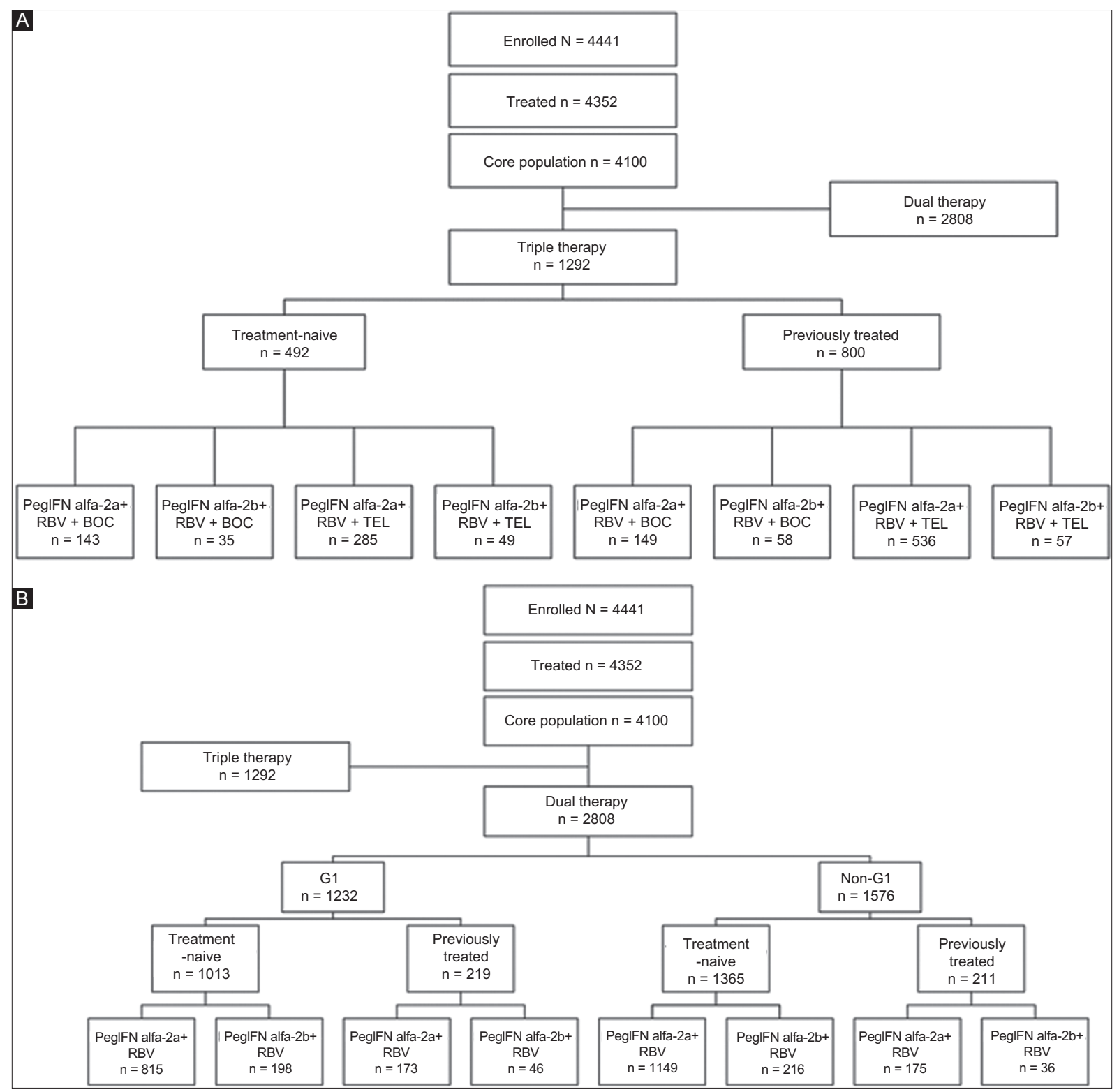

Figure 1 Disposition of treatment-näve and previously treated patients with chronic hepatitis $C$ who were enrolled and treated with boceprevir (BOC)- or telaprevir (TEL)-based triple therapy or dual peginterferon (PegIFN) alfa-2a or alfa-2b plus ribavirin (RBV). (A) Patients assigned to BOC- or TEL-based triple therapy. (B) Patients assigned to dual PegIFN alfa-2a or alfa-2b plus ribavirin therapy

was the most frequently cited reason for peginterferon alfa dose modifications (Table 7). Approximately $40 \%$ of patients in each treatment group experienced a hemoglobin level $<100 \mathrm{~g} / \mathrm{dL}$ during therapy and approximately $40 \%$ to $50 \%$ of patients experienced a platelet level $<100 \times 10^{9} / \mathrm{L}$ at some point during treatment (Table 7). Hepatic failure was rare, being reported as an SAE in two patients assigned to telaprevir plus peginterferon alfa- $2 \mathrm{a} /$ ribavirin and as a non-serious $\mathrm{AE}$ in two additional patients (one assigned to boceprevir plus peginterferon alfa-2a/ribavirin, and one assigned to telaprevir plus peginterferon alfa-2a/ribavirin). Infections were reported as SAEs in 11 of 385 (2.9\%) patients assigned to boceprevir- based triple therapy and in 34 of 907 (3.7\%) patients assigned to telaprevir-based triple therapy. A total of 11 patients assigned to boceprevir- or telaprevir-based triple therapy died during the study; seven of the deaths were unrelated to $\mathrm{CHC}$ or treatment. Two deaths were considered to be related to $\mathrm{CHC}$ and to the study drug in the opinion of the investigator; both patients had been assigned to telaprevir plus peginterferon alfa-2a (one death was attributed to esophageal variceal hemorrhage and the other to hepatic failure). Two additional deaths from liver cancer, both in patients assigned to telaprevir plus peginterferon alfa-2a, were considered to be related to $\mathrm{CHC}$, but not to the study drug. 
Table 3 Patient disposition

\begin{tabular}{|c|c|c|c|c|c|c|}
\hline $\begin{array}{l}\text { Patient disposition, } \\
\mathrm{n}(\%)\end{array}$ & $\begin{array}{c}\text { Boceprevir plus } \\
\text { PegIFN alfa- } \\
\text { 2a/RBV }\end{array}$ & $\begin{array}{c}\text { Boceprevir plus } \\
\text { PegIFN alfa- } \\
\text { 2b/RBV }\end{array}$ & $\begin{array}{l}\text { Telaprevir plus } \\
\text { PegIFN alfa- } \\
\text { 2a/RBV }\end{array}$ & $\begin{array}{c}\text { Telaprevir plus } \\
\text { PegIFN alfa- } \\
\text { 2b/RBV }\end{array}$ & $\begin{array}{c}\text { PegIFN alfa- } \\
\text { 2a/RBV }\end{array}$ & $\begin{array}{l}\text { PegIFN alfa- } \\
\text { 2b/RBV }\end{array}$ \\
\hline \multicolumn{7}{|l|}{ Treatment-naïve } \\
\hline HCV genotype 1 & $\mathrm{~N}=143$ & $\mathrm{~N}=35$ & $\mathrm{~N}=285$ & $\mathrm{~N}=29$ & $\mathrm{~N}=815$ & $N=198$ \\
\hline $\begin{array}{l}\text { Completed } 12 \text {-week } \\
\text { treatment }\end{array}$ & $130(90.9)$ & $34(97.1)$ & $259(90.9)$ & $26(89.7)$ & $776(95.2)$ & $178(89.9)$ \\
\hline $\begin{array}{l}\text { Completed 24-week } \\
\text { follow up }\end{array}$ & $97(67.8)$ & $24(68.6)$ & $215(75.4)$ & $19(65.5)$ & $556(68.2)$ & $121(61.1)$ \\
\hline HCV genotype non-1 & - & - & - & - & $\mathrm{N}=1149$ & $\mathrm{~N}=216$ \\
\hline $\begin{array}{l}\text { Completed } 12 \text {-week } \\
\text { treatment }\end{array}$ & & & & & $1089(94.8)$ & $202(93.5)$ \\
\hline $\begin{array}{l}\text { Completed 24-week } \\
\text { follow up }\end{array}$ & & & & & $834(72.6)$ & $173(80.1)$ \\
\hline Previously treated & $n=149$ & $\mathrm{n}=58$ & $n=536$ & $\mathrm{n}=\mathbf{5 7}$ & $\mathrm{n}=348$ & $\mathrm{n}=82$ \\
\hline $\begin{array}{l}\text { Completed } 12 \text {-week } \\
\text { treatment }\end{array}$ & $135(90.6)$ & $50(86.2)$ & $481(89.7)$ & $50(87.7)$ & $315(90.5)$ & $75(91.5)$ \\
\hline $\begin{array}{l}\text { Completed } 24 \text {-week } \\
\text { follow up }\end{array}$ & $95(63.8)$ & $36(62.1)$ & $395(73.7)$ & $35(61.4)$ & $200(57.5)$ & $37(45.1)$ \\
\hline
\end{tabular}

Table 4 Reasons for withdrawal from treatment with peginterferon

\begin{tabular}{|c|c|c|c|c|c|c|}
\hline $\begin{array}{l}\text { Reason for withdrawal, } \\
\text { n (\%) }\end{array}$ & $\begin{array}{c}\text { Boceprevir plus } \\
\text { PegIFN alfa-2a/ } \\
\text { RBV }\end{array}$ & $\begin{array}{c}\text { Boceprevir plus } \\
\text { PegIFN alfa-2b/ } \\
\text { RBV }\end{array}$ & $\begin{array}{c}\text { Telaprevir plus } \\
\text { PegIFN alfa-2a/ } \\
\text { RBV }\end{array}$ & $\begin{array}{c}\text { Telaprevir plus } \\
\text { PegIFN alfa-2b/ } \\
\text { RBV }\end{array}$ & $\begin{array}{c}\text { PegIFN } \\
\text { alfa-2a/RBV }\end{array}$ & $\begin{array}{c}\text { PegIFN } \\
\text { alfa-2b/RBV }\end{array}$ \\
\hline Treatment-naïve & $\mathrm{N}=143$ & $\mathrm{~N}=35$ & $\mathrm{~N}=285$ & $\mathrm{~N}=29$ & $\mathrm{~N}=1964$ & $\mathrm{~N}=414$ \\
\hline Adverse event & $20(14.0)$ & $1(2.9)$ & $40(14.0)$ & $3(10.3)$ & $111(5.7)$ & $37(8.9)$ \\
\hline Insufficient response & $14(9.8)$ & $4(11.4)$ & $10(3.5)$ & $4(13.8)$ & $196(10.0)$ & $53(12.8)$ \\
\hline Good response & $5(3.5)$ & $1(2.9)$ & $12(4.2)$ & $1(3.4)$ & $59(3.0)$ & $14(3.4)$ \\
\hline $\begin{array}{l}\text { Refused treatment, } \\
\text { withdrew consent, or } \\
\text { did not cooperate }\end{array}$ & $5(3.5)$ & $2(5.7)$ & $17(6.0)$ & $4(13.8)$ & $102(5.2)$ & $20(4.8)$ \\
\hline Failure to return & $7(4.9)$ & $0(0)$ & $6(2.1)$ & $1(3.4)$ & $92(4.7)$ & $16(3.9)$ \\
\hline $\begin{array}{l}\text { Administrative or } \\
\text { other reason }\end{array}$ & $1(0.7)$ & $0(0)$ & $2(0.7)$ & $0(0)$ & $11(0.6)$ & $4(1.0)$ \\
\hline Death & $0(0)$ & $0(0)$ & $0(0)$ & $0(0)$ & $1(<0.1)$ & $1(0.2)$ \\
\hline Previously treated & $\mathrm{N}=149$ & $\mathrm{~N}=58$ & $\mathrm{~N}=536$ & $\mathrm{~N}=57$ & $\mathrm{~N}=348$ & $\mathrm{~N}=82$ \\
\hline Adverse event & $17(11.4)$ & $8(13.8)$ & $48(9.0)$ & $5(8.8)$ & $34(9.8)$ & $6(7.3)$ \\
\hline Insufficient response & $36(24.2)$ & $12(20.7)$ & $85(15.9)$ & $6(10.5)$ & $76(21.8)$ & $23(28.0)$ \\
\hline Good response & $1(0.7)$ & $1(1.7)$ & $24(4.5)$ & $4(7.0)$ & $4(1.1)$ & $0(0)$ \\
\hline $\begin{array}{l}\text { Refused treatment, } \\
\text { withdrew consent, or } \\
\text { did not cooperate }\end{array}$ & $2(1.3)$ & $3(5.2)$ & $21(3.9)$ & $2(3.5)$ & $22(6.3)$ & $3(3.7)$ \\
\hline Failure to return & $4(2.7)$ & $1(1.7)$ & $13(2.4)$ & $5(8.8)$ & $15(4.3)$ & $3(3.7)$ \\
\hline $\begin{array}{l}\text { Administrative or } \\
\text { other reason }\end{array}$ & $1(0.7)$ & $1(1.7)$ & $11(2.1)$ & $0(0)$ & $2(0.6)$ & $2(2.4)$ \\
\hline Death & $0(0)$ & $0(0)$ & $1(0.2)$ & $0(0)$ & $0(0)$ & $0(0)$ \\
\hline
\end{tabular}

PegIFN, peginterferon; $R B V$, ribavirin 
A

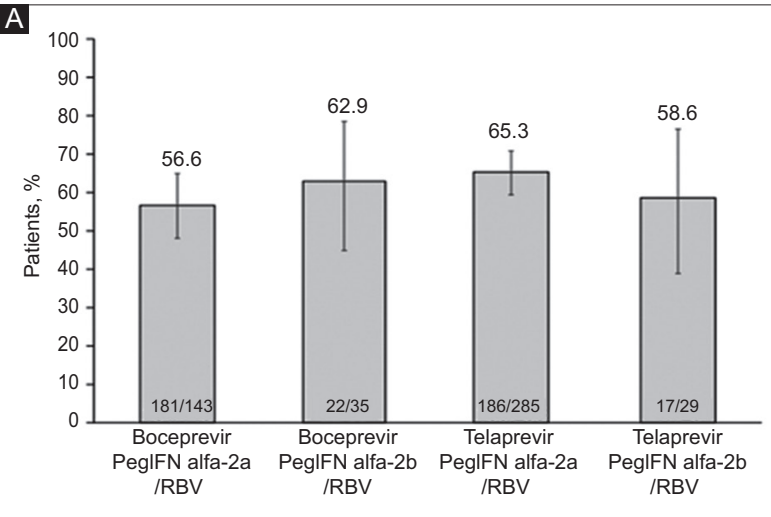

C

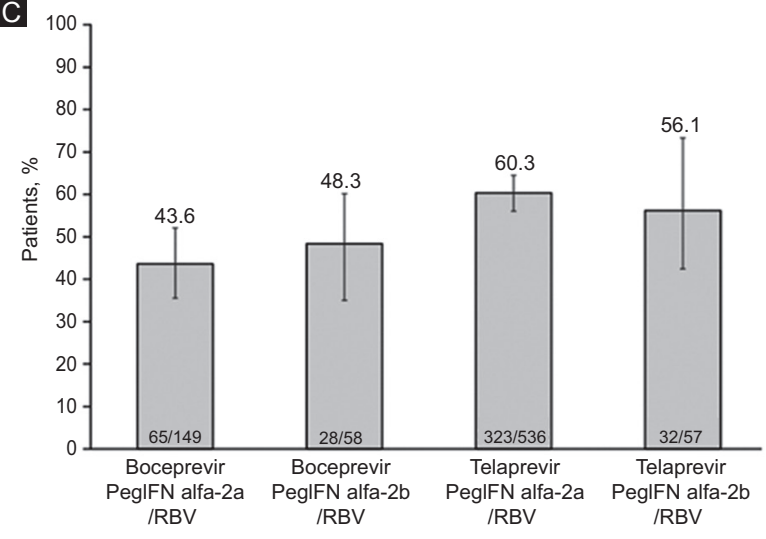

B

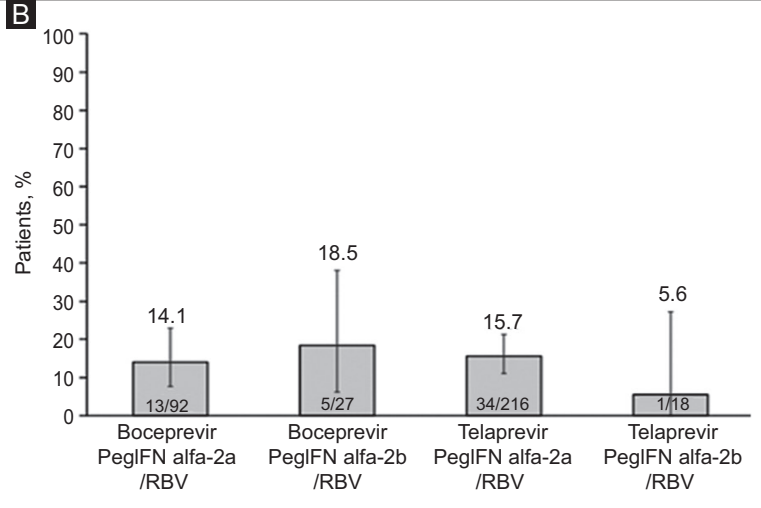

D 100

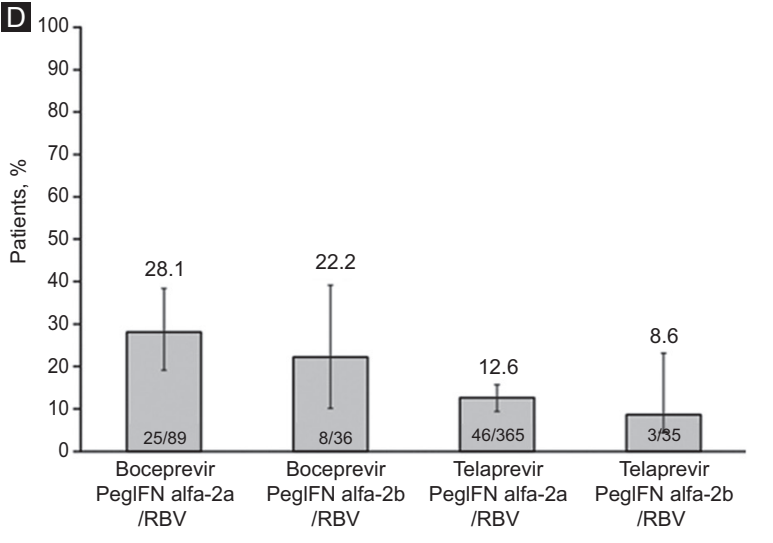

Figure 2 SVR12 and relapse rates for patients receiving boceprevir- and telaprevir-based triple therapy. (A) SVR12 rates in treatment- naïve patients including 95\% confidence intervals. (B) Relapse rates in treatment-naïve patients including 95\% confidence intervals. (C) SVR12 rates in previously treated patients including $95 \%$ confidence intervals. (D) Relapse rates in previously treated patients including $95 \%$ confidence intervals PegIFN, peginterferon; RBV, ribavirin; SVR12, sustained virological response 12 weeks after the end of treatment

\section{VR and safety in patients receiving dual therapy}

Among treatment-naïve patients assigned to dual therapy with peginterferon alfa-2a/ribavirin and peginterferon alfa- $2 \mathrm{~b} /$ ribavirin, SVR 12 rates were $49.2 \%$ and $41.9 \%$, respectively, in patients with G1 infection, $75.7 \%$ and $83.3 \%$ in patients with G2 infection, $65.9 \%$ and $65.9 \%$ in patients with G3 infection, and $49.7 \%$ and $51.1 \%$ in patients with G4 infection (Table 8 ). SVR12 rates are shown by HCV genotype and baseline characteristic in Table 9.

Among previously treated patients assigned to dual therapy with peginterferon alfa-2a/ribavirin and peginterferon alfa- $2 \mathrm{~b} /$ ribavirin, overall SVR12 rates were $35.6 \%$ (124/348) and 25.6\% $(21 / 82)$, respectively.

The overall safety profile of dual therapy is presented in Table 10. Among patients assigned to peginterferon alfa-2a/ ribavirin and peginterferon alfa- $2 \mathrm{~b} /$ ribavirin, the incidence of AEs was $60.3 \%$ and $65.7 \%$ respectively, and the incidence of SAEs was $6.4 \%$ and $6.7 \%$, respectively. The most commonly reported AE was anemia (in $19.7 \%$ and $28.6 \%$ of patients, respectively). Safety-related dosage modifications were required more frequently for ribavirin than for the peginterferon alfa component of the respective regimens and the most frequently cited reason for ribavirin dose reductions was anemia (in $13.6 \%$ and $20.4 \%$ of patients assigned to peginterferon alfa-2a/ ribavirin and peginterferon alfa-2b/ribavirin, respectively) (Table 10).

Hepatic failure was reported as an SAE in three patients (two recipients of peginterferon alfa-2a and 1 recipient of peginterferon alfa-2b). Infections were reported as SAEs in 35 of $2312(1.5 \%)$ patients assigned peginterferon alfa2a plus ribavirin and in 8 of $496(1.6 \%)$ patients assigned to peginterferon alfa-2b plus ribavirin. A total of nine patients assigned to dual therapy died, including 6 deaths in patients treated with peginterferon alfa-2a/ribavirin and 3 deaths in patients treated with peginterferon alfa-2b/ribavirin. All were unrelated to treatment in the opinion of the investigator, two were considered to be related to $\mathrm{CHC}$ (septic shock in a patient treated with peginterferon alfa-2a/ribavirin, and gastrointestinal hemorrhage in a patient treated with peginterferon alfa-2b/ribavirin), and for two patients no assessment of the relationship to $\mathrm{CHC}$ was provided.

\section{Discussion}

With the advent of second-generation DAAs, telaprevir and boceprevir have been withdrawn from the US market and PI-based triple therapy and dual peginterferon alfa/ 
Table 5 Virological response (VR), relapse and breakthrough rates - triple therapy

\begin{tabular}{|c|c|c|c|c|}
\hline \multirow[t]{2}{*}{ Event } & \multicolumn{4}{|c|}{ Response rate $\mathrm{n},(\% ; 95 \% \mathrm{CI})$} \\
\hline & $\begin{array}{c}\text { Boceprevir plus } \\
\text { PegIFN alfa-2a/RBV }\end{array}$ & $\begin{array}{c}\text { Boceprevir plus } \\
\text { PegIFN alfa-2b/RBV }\end{array}$ & $\begin{array}{c}\text { Telaprevir plus } \\
\text { PegIFN alfa-2a/RBV }\end{array}$ & $\begin{array}{c}\text { Telaprevir plus } \\
\text { PegIFN alfa-2b/RBV }\end{array}$ \\
\hline Treatment-naïve & $\mathrm{N}=143$ & $\mathrm{~N}=35$ & $\mathrm{~N}=\mathbf{2 8 5}$ & $\mathrm{N}=29$ \\
\hline eRVR & $67(46.9 ; 38.5-55.4)$ & $15(42.9 ; 26.3-60.6)$ & $122(42.8 ; 37.0-48.8)$ & $11(37.9 ; 20.7-57.7)$ \\
\hline EoT VR & $103(72.0 ; 63.9-79.2)$ & $29(82.9 ; 66.4-93.4)$ & $228(80.0 ; 74.9-840.5)$ & $19(65.5 ; 45.7-82.1)$ \\
\hline SVR12 & $81(56.6 ; 48.1-64.9)$ & $22(62.9 ; 44.9-78.5)$ & $186(65.3 ; 59.4-70.8)$ & $17(58.6 ; 38.9-76.5)$ \\
\hline SVR24 & $75(52.4 ; 43.9-60.9)$ & $20(57.1 ; 39.4-73.7)$ & $169(59.3 ; 53.3-65.1)$ & $15(51.7 ; 32.5-70.6)$ \\
\hline Relapse & $13 / 92(14.1 ; 7.7-23.0)$ & $5 / 27(18.5 ; 6.3-38.1)$ & $34 / 216(15.7 ; 11.2-21.3)$ & $1 / 18(5.6 ; 0.1-27.3)$ \\
\hline Breakthrough/rebound & $11 / 134(8.2 ; 4.2-14.2)$ & $5 / 35(14.3 ; 4.8-30.3)$ & $22 / 272(8.1 ; 5.1-12.0)$ & $6 / 26(23.1 ; 9.0-43.6)$ \\
\hline Previously treated & $n=149$ & $\mathrm{n}=58$ & $n=536$ & $\mathrm{n}=\mathbf{5 7}$ \\
\hline $\mathrm{eRVR}^{\star}$ & $43(28.9 ; 21.7-36.8)$ & $15(25.9 ; 15.3-39.0)$ & $252(47.0 ; 42.7-51.3)$ & $30(52.6 ; 39.0-66.0)$ \\
\hline EoT VR & $93(62.4 ; 54.1-70.2)$ & $38(65.5 ; 51.9-77.5)$ & $387(72.2 ; 68.2-76.0)$ & $38(66.7 ; 52.9-78.6)$ \\
\hline SVR12 & $65(43.6 ; 35.5-52.0)$ & $28(48.3 ; 35.0-61.8)$ & $323(60.3 ; 56.0-64.4)$ & $32(56.1 ; 42.4-69.3)$ \\
\hline SVR24 & $61(40.9 ; 33.0-49.3)$ & $27(46.6 ; 33.3-60.1)$ & $305(56.9 ; 52.6-61.1)$ & $26(45.6 ; 32.4-59.3)$ \\
\hline Relapse & $25 / 89(28.1 ; 19.1-38.6)$ & $8 / 36(22.2 ; 10.1-39.2)$ & $46 / 365$ (12.6; 9.4-16.5) & $3 / 35(8.6 ; 1.8-23.1)$ \\
\hline Breakthrough/rebound & $13 / 141(9.2 ; 5.0-15.3)$ & $9 / 53(17.0 ; 8.1-29.8)$ & $96 / 513(18.7 ; 15.4-22.4)$ & $10 / 48(20.8 ; 10.5-35.0)$ \\
\hline
\end{tabular}

VR was defined as undetectable HCV RNA as assessed by a test with lower limit of detection $\leq 50 \mathrm{IU} / \mathrm{mL}$, ${ }^{*}$ RVR was defined differently for patients receiving telaprevir (VR at weeks 4 and 12) and boceprevir (VR at weeks 8 and 24), Relapse was defined as non-response at the last HCV RNA assessment during the treatment-free follow-up period in patients with an EoT response. Patients were included in the calculation of relapse if they had an EoT response and an available HCV RNA measurement for SVR12 or an earlier follow-up assessment showing non-response during the treatment-free follow-up period (i.e., patients lost to follow up after EoT were not included in the calculation of relapse), Breakthrough/rebound was defined as non-response during the treatment period in patients with a previous response or increase in HCV RNA by $\geq 1 \log 10$ during the treatment period versus the lowest value previously recorded during the treatment period. Patients were included in the calculation of breakthrough/rebound if they had $\geq 2$ HCV RNA measurements during treatment (which could include imputation of EoT response)

CI, confidence interval; EoT, end of treatment; eRVR, extended rapid virological response; PegIFN, peginterferon; RBV, ribavirin; SVR12, sustained virological response at least 12 weeks after the end of treatment ( $\geq 70$ days after day of last dose); SVR24, sustained virological response at least 24 weeks after end of treatment ( $\geq 140$ days after day of last dose)

ribavirin therapy are no longer recommended in treatment guidelines as a preferred treatment for patients infected with HCV G1 [10-12]. However, availability and cost restrictions mean that peginterferon-based regimens may continue in some countries, as is explicitly recognized by the European and Asian guidelines [11,12]. It therefore remains important to understand the efficacy and safety of peginterferon-based regimens in real-world settings, and the PegBase observational study provides data to this end.

The efficacy of first-generation PI-based triple therapy in PegBase was somewhat lower than that reported in phase III studies. The range of SVR12 rates achieved in treatmentnaïve patients with boceprevir-based triple therapy in the PegBase study (57-63\%) overlaps with that obtained in the phase III trial (SPRINT-2, 63-66\%) [7]. SVR12 rates achieved with telaprevir-based triple therapy in PegBase (59-65\%) are below those observed with the approved regimen in ADVANCE (75\%) [6] and ILLUMINATE (72\%) [14]. These results are likely to be a reflection of differences between the highly restrictive criteria used to select patients for registration studies, the heterogeneous characteristics of patients encountered in routine clinical practice, and differences in the extent of monitoring and follow up between registration trials and real-world studies. For example, a higher proportion of patients with fibrosis assessments had transition to cirrhosis or had cirrhosis in PegBase (>30\%) than in phase III trials of boceprevir (7-9\%) or telaprevir (20\%).

Likewise, in previously treated patients, overall SVR12 rates in PegBase were 44-48\% in patients assigned to boceprevir-based triple therapy, compared with 59-66\% in RESPOND-2 [8], 56-60\% in patients assigned to telaprevir-based triple therapy and $64 \%$ with the approved regimen in REALIZE [9]. Responses in previously treated patients are largely a reflection of the type of prior therapy, for example, interferon monotherapy or combination therapy with ribavirin, and the nature of the prior response to interferon-based therapy, with generally higher SVR rates in relapsers than in non-responders [15-17]. In RESPOND-2, approximately two-thirds of patients had a prior relapse and approximately one-third of patients had a prior non-response to peginterferon-based therapy [8]. Likewise, more than half of the patients in REALIZE (53\%) had a previous relapse and $28 \%$ had a previous non-response to peginterferonbased therapy [9]. In contrast, almost half of the previously treated patients enrolled in the present study were prior nonresponders, while only one-third of them had experienced a prior relapse. In addition, some previously treated patients had received conventional interferon monotherapy or peginterferon monotherapy. Thus, differences in response rates between 
Table 6 Subgroup analysis by baseline characteristics of SVR12 in HCV G1 patients receiving triple therapy

\begin{tabular}{|c|c|c|c|c|}
\hline \multirow[t]{2}{*}{ Baseline characteristic } & \multicolumn{4}{|c|}{ Patients with SVR12, n (\%) } \\
\hline & $\begin{array}{l}\text { Boceprevir plus } \\
\text { egIFN alfa-2a/RBV }\end{array}$ & $\begin{array}{c}\text { Boceprevir plus } \\
\text { PegIFN alfa-2b/RBV }\end{array}$ & $\begin{array}{c}\text { Telaprevir plus } \\
\text { PegIFN alfa-2a/RBV }\end{array}$ & $\begin{array}{c}\text { Telaprevir plus } \\
\text { PegIFN alfa-2b/RBV }\end{array}$ \\
\hline Treatment-naïve & $\mathrm{N}=143$ & $\mathrm{~N}=35$ & $\mathrm{~N}=\mathbf{2 8 5}$ & $\mathrm{N}=\mathbf{2 9}$ \\
\hline Overall & $81(56.6)$ & $22(62.9)$ & $186(65.3)$ & $17(58.6)$ \\
\hline \multicolumn{5}{|l|}{ Fibrosis status } \\
\hline Cirrhosis & $24 / 55(43.6)$ & $6 / 12(50.0)$ & $53 / 98(54.1)$ & $10 / 18(55.6)$ \\
\hline No cirrhosis & $57 / 88(64.8)$ & $16 / 23(69.6)$ & $133 / 187(71.1)$ & $7 / 11(63.6)$ \\
\hline \multicolumn{5}{|c|}{ IL28B rs12979860 host genotype } \\
\hline $\mathrm{CC}$ & $18 / 25(72.0)$ & $4 / 5(80.0)$ & $26 / 29(89.7)$ & $2 / 3(66.7)$ \\
\hline $\mathrm{CT}$ & $22 / 39(56.4)$ & $6 / 8(75.0)$ & $51 / 82(62.2)$ & $7 / 12(58.3)$ \\
\hline $\mathrm{TT}$ & $10 / 16(62.5)$ & $0 / 2(0.0)$ & $22 / 32(68.8)$ & $1 / 4(25.0)$ \\
\hline Unknown & $31 / 63(49.2)$ & $12 / 20(60.0)$ & $87 / 142(61.3)$ & $7 / 10(70.0)$ \\
\hline \multicolumn{5}{|l|}{ HCV RNA, IU/mL } \\
\hline$\leq 400,000$ & $15 / 32(46.9)$ & $6 / 7(85.7)$ & $51 / 73(69.9)$ & $6 / 9(66.7)$ \\
\hline$>400,000$ & $66 / 111(59.5)$ & $15 / 27(55.6)$ & $135 / 212(63.7)$ & $11 / 20(55.0)$ \\
\hline Missing & - & $1 / 1(100)$ & - & - \\
\hline Previously treated & $\mathrm{n}=149$ & $\mathrm{n}=\mathbf{5 8}$ & $\mathrm{n}=536$ & $\mathrm{n}=\mathbf{5 7}$ \\
\hline Overall & $65(43.6)$ & $28(48.3)$ & $323(60.3)$ & $32(56.1)$ \\
\hline \multicolumn{5}{|l|}{ Fibrosis status } \\
\hline Cirrhosis & $27 / 83(32.5)$ & $12 / 29(41.4)$ & $124 / 243(51.0)$ & $14 / 25(56.0)$ \\
\hline No cirrhosis & $38 / 66(57.6)$ & $16 / 29(55.2)$ & $199 / 293(67.9)$ & $18 / 32(56.3)$ \\
\hline \multicolumn{5}{|c|}{ IL28B rs 12979860 host genotype ${ }^{*}$} \\
\hline $\mathrm{CC}$ & $4 / 10(40.0)$ & $4 / 5(80.0)$ & $33 / 43(76.7)$ & $3 / 5(60.0)$ \\
\hline $\mathrm{CT}$ & $24 / 51(47.1)$ & $11 / 25(44.0)$ & $102 / 179(57.0)$ & $15 / 27(55.6)$ \\
\hline TT & $7 / 21(33.3)$ & $4 / 7(57.1)$ & $36 / 65(55.4)$ & $4 / 10(40.0)$ \\
\hline Unknown & $30 / 67(44.8)$ & $9 / 21(42.9)$ & $151 / 248(60.9)$ & $10 / 15(66.7)$ \\
\hline \multicolumn{5}{|l|}{ HCV RNA, IU/mL } \\
\hline$\leq 400,000$ & $19 / 36(52.8)$ & $8 / 14(57.1)$ & $85 / 117(72.6)$ & $12 / 16(75.0)$ \\
\hline$>400,000$ & $46 / 113(40.7)$ & 20/44 (45.5) & $236 / 417(56.6)$ & $20 / 41(48.8)$ \\
\hline Missing & - & - & $2 / 2(100.0)$ & - \\
\hline \multicolumn{5}{|l|}{ Prior response, $\mathrm{n}(\%)$} \\
\hline Relapse & $30 / 44(68.2)$ & $13 / 19(68.4)$ & $140 / 177(79.1)$ & $14 / 27(51.9)$ \\
\hline Breakthrough & $1 / 5(20.0)$ & $2 / 3(66.7)$ & $22 / 37(59.5)$ & $1 / 2(50.0)$ \\
\hline Non-response & 29/82 (35.4) & $9 / 28(32.1)$ & $117 / 248(47.2)$ & $11 / 17(64.7)$ \\
\hline Other & $4 / 17(23.5)$ & $3 / 7(42.9)$ & $39 / 68(57.4)$ & $6 / 10(60.0)$ \\
\hline Prior response unknown & $1 / 1(100)$ & $1 / 1(100)$ & $5 / 6(83.3)$ & $0 / 1(0)$ \\
\hline
\end{tabular}

$\overline{\text { SVR12 }}$ = percentage of patients with undetectable HCV RNA (to a test with lower limit of detection $\leq 50 \mathrm{IU} / \mathrm{mL}$ ) at 12 weeks after completion of the treatment period (at least 70 days after day of last treatment), ${ }^{*}$ Host genotype was unknown in 235 treatment-naïve patients and 351 Previously treated patients. One previously treated patient was negative for rs 12979860

HCV, hepatitis C virus; PegIFN, peginterferon; RBV, ribavirin; SVR, sustained virological response

PegBase and phase III studies reflect not only differences in baseline characteristics, but also differences in the proportion of patients with prior relapse or non-response and in the intensity of the previous treatment regimen.
Predictors of good VR include absence of cirrhosis, homozygosity for the IL28B rs 12979860 C allele and low viral load [18]. In the PegBase study, patients without transition to cirrhosis or cirrhosis had better virological outcomes than 
Table 7 Safety outcomes in patients with HCV genotype 1 mono-infection receiving triple therapy (includes treatment-naive and-previously treated patients)

\begin{tabular}{|c|c|c|c|c|}
\hline Event & $\begin{array}{c}\text { Boceprevir plus } \\
\text { PegIFN alfa- } \\
\text { 2a/RBV }\end{array}$ & $\begin{array}{c}\text { Boceprevir plus } \\
\text { PegIFN alfa- } \\
\text { 2b/RBV }\end{array}$ & $\begin{array}{l}\text { Telaprevir plus } \\
\text { PegIFN alfa- } \\
\text { 2a/RBV }\end{array}$ & $\begin{array}{c}\text { Telaprevir plus } \\
\text { PegIFN alfa- } \\
\text { 2b/RBV }\end{array}$ \\
\hline & $\mathrm{N}=292$ & $\mathrm{~N}=93$ & $\mathrm{~N}=821$ & $\mathrm{~N}=86$ \\
\hline Patients with $\geq 1 \mathrm{AE}, \mathrm{n}(\%)$ & $224(76.7)$ & $82(88.2)$ & $745(90.7)$ & $75(87.2)$ \\
\hline Patients with $\geq 1$ SAE, $n$ (\%) & $43(14.7)$ & $9(9.7)$ & $163(19.9)$ & $4(4.7)$ \\
\hline Death & $2(0.7)$ & $1(1.1)$ & $8(1.0)$ & 0 \\
\hline \multicolumn{5}{|l|}{ Incidence of individual AEs*, n (\%) } \\
\hline Anemia, n (\%) & $120(41.1)$ & $45(48.4)$ & $398(48.5)$ & $44(51.2)$ \\
\hline Neutropenia & $39(13.4)$ & $25(26.9)$ & $86(10.5)$ & $24(27.9)$ \\
\hline Asthenia & $53(18.2)$ & $23(24.7)$ & $246(30.0)$ & $9(10.5)$ \\
\hline Pruritus & $37(12.7)$ & $16(17.2)$ & $240(29.2)$ & $10(11.6)$ \\
\hline Thrombocytopenia & $33(11.3)$ & $6(6.5)$ & $98(11.9)$ & $23(26.7)$ \\
\hline Leukopenia & $10(3.4)$ & $6(6.5)$ & $60(7.3)$ & $31(36.0)$ \\
\hline Nausea & $36(12.3)$ & $24(25.8)$ & $135(16.4)$ & $11(12.8)$ \\
\hline Fatigue & $68(23.3)$ & $23(24.7)$ & $172(21.0)$ & $13(15.1)$ \\
\hline Rash & $26(8.9)$ & $9(9.7)$ & $161(19.6)$ & $10(11.6)$ \\
\hline Influenza-like illness & $31(10.6)$ & $17(18.3)$ & $97(11.8)$ & $7(8.1)$ \\
\hline Decreased appetite & $25(8.6)$ & $16(17.2)$ & $118(14.4)$ & $8(9.3)$ \\
\hline Headache & $33(11.3)$ & $15(16.1)$ & $94(11.4)$ & $5(5.8)$ \\
\hline Dysgeusia & $33(11.3)$ & $14(15.1)$ & $40(4.9)$ & $1(1.2)$ \\
\hline Hemoglobin increased & $1(0.3)$ & 0 & $16(1.9)$ & $13(15.1)$ \\
\hline Insomnia & $18(6.2)$ & $7(7.5)$ & $100(12.2)$ & $4(4.7)$ \\
\hline Diarrhea & $17(5.8)$ & $11(11.8)$ & $79(9.6)$ & $5(5.8)$ \\
\hline Pyrexia & $14(4.8)$ & $11(11.8)$ & $36(4.4)$ & $4(4.7)$ \\
\hline Alopecia & $14(4.8)$ & $10(10.8)$ & $51(6.2)$ & $2(2.3)$ \\
\hline Cough & $23(7.9)$ & $10(10.8)$ & $72(8.8)$ & $1(1.2)$ \\
\hline Dry skin & $22(7.5)$ & $10(10.8)$ & $66(8.0)$ & $3(3.5)$ \\
\hline Peginterferon dose modification $\ddagger$ n (\%) & $49(16.8)$ & $18(19.4)$ & $104(12.7)$ & $9(10.5)$ \\
\hline Neutropenia & $31(10.6)$ & $10(10.8)$ & $24(2.9)$ & $5(5.8)$ \\
\hline Thrombocytopenia & $9(3.1)$ & $1(1.1)$ & $37(4.5)$ & $3(3.5)$ \\
\hline Anemia & $3(1.0)$ & $2(2.2)$ & $14(1.7)$ & 0 \\
\hline Other & $8(2.7)$ & $7(7.5)$ & $36(4.4)$ & $1(1.2)$ \\
\hline Ribavirin dose modification ${ }^{\ddagger}, \mathrm{n}(\%)$ & $125(42.8)$ & $40(43.0)$ & $381(46.4)$ & $31(36.0)$ \\
\hline Anemia & $115(39.4)$ & $37(39.8)$ & $336(40.9)$ & $29(33.7)$ \\
\hline Other & $14(4.8)$ & $8(8.6)$ & $75(9.1)$ & $2(2.3)$ \\
\hline \multicolumn{5}{|c|}{ Laboratory abnormalities, lowest value after BL, n (\%) } \\
\hline Hemoglobin $<100 \mathrm{~g} / \mathrm{L}$ & $121(41.6)$ & $36(39.1)$ & $357(43.8)$ & $36(43.4)$ \\
\hline Platelets $<100 \times 10^{9} / \mathrm{L}$ & $145(50.0)$ & $42(45.7)$ & $400(49.1)$ & $31(37.3)$ \\
\hline Neutrophils $<2.0 \times 10^{9} / \mathrm{L}$ & $262(90.7)$ & $85(92.4)$ & 707 (87.4) & $60(73.2)$ \\
\hline
\end{tabular}

${ }^{*}$ Incidence $\geq 10 \%$ in at least one of the treatment groups, ${ }^{\dagger}$ Total number of deaths regardless of relation to treatment in the opinion of the investigator, ${ }^{*}$ Because of adverse event or laboratory abnormality

$A E$, adverse event; $B L$, baseline; $H C V$, hepatitis $C$ virus; PegIFN, peginterferon; $R B V$, ribavirin; SAE, serious adverse event 


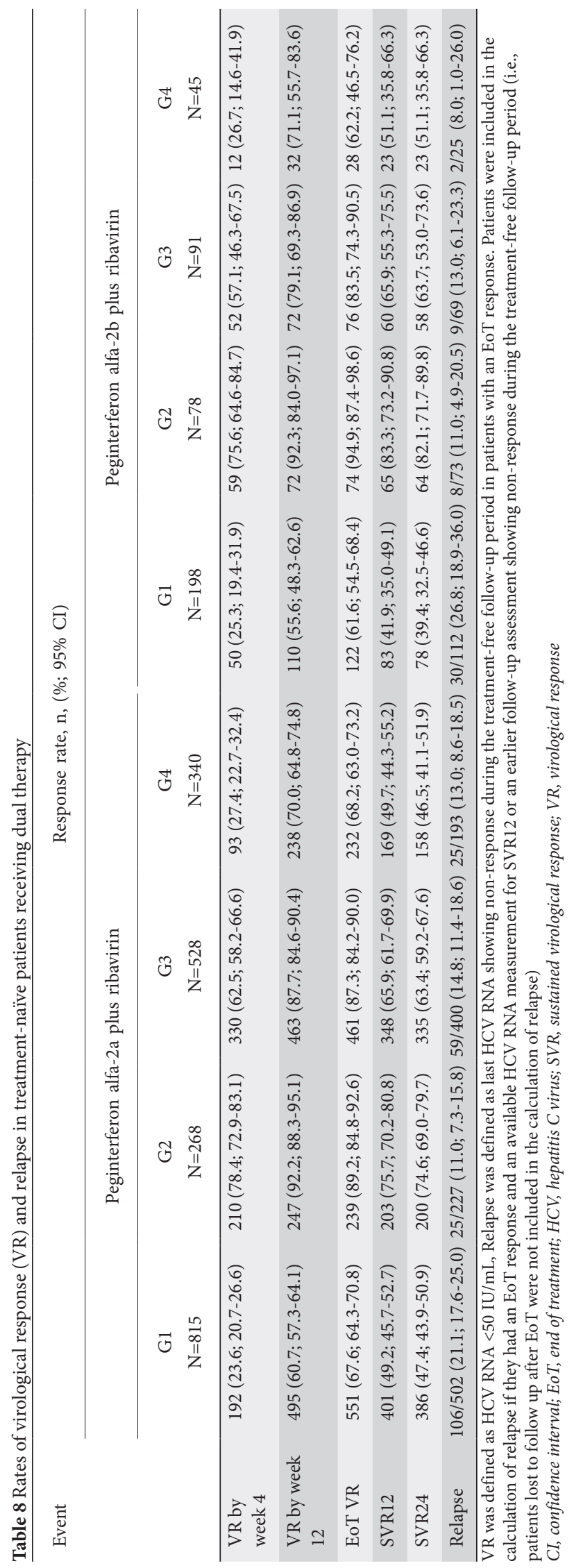

patients with transition to cirrhosis or cirrhosis, and higher response rates were seen in patients homozygous for the IL28B rs12979860 C allele than in patients heterozygous for the C and $\mathrm{T}$ alleles or in patients homozygous for the $\mathrm{T}$ allele. There was little difference in treatment response between treatmentnaïve patients with high or low viral loads; however, previously treated patients with low viral loads responded better than those with high viral loads.

The spectrum of AEs observed in PegBase is consistent with that reported in phase III studies of boceprevir- and telaprevirbased triple therapy [6-9]. The lower incidence of certain AEs, such as rash, in PegBase (approximately 16\%) than in phase III trials $(>30 \%)$ is possibly a reflection of closer patient monitoring in registration studies.

The efficacy of first-generation PI-based triple therapy in PegBase is consistent with other "real-world" studies in that they also report lower SVR rates than those in registration studies [19-22]. For example, in the German PAN cohort study [23], SVR12 rates of 55\% (boceprevir) and 63\% (telaprevir) were obtained in treatment-naïve patients and $51 \%$ (boceprevir) and 68\% (telaprevir) in previously treated patients. The Kaiser Permanente Medical Care Program in Northern California reported SVR rates of 53\% and 56\% with boceprevir- and telaprevir-based triple therapy [21]. SVR rates were also lower in previously treated patients with cirrhosis who received first-generation triple therapy in the French CUPIC study [20]. The CUPIC study is noteworthy in that it reported comparatively high rates of SAEs (50\%) and hepatic decompensation $(8 \%)$, and deaths were associated with severe infections, perhaps as a result of the inclusion of patients with contraindications for the triple-therapy regimens. The lower rates of SAEs, hepatic failure and the absence of a link between serious infection and deaths in PegBase suggests that contraindications were observed when selecting patients for PegBase.

The efficacy and safety profile of dual peginterferon alfa/ ribavirin therapy in PegBase is similar to that reported in previous randomized controlled studies and in other large real-world studies [24-27]. The place of dual therapy in the treatment of $\mathrm{CHC}$ has continued to diminish since the PegBase study was initiated. In particular, dual therapy is no longer suitable in patients with access to DAAs, because of the lower efficacy and longer duration of treatment [10-12].

No firm conclusions can be drawn regarding the comparative efficacy or safety of boceprevir and telaprevir or of peginterferon alfa- $2 a$ and peginterferon alfa- $2 b$ on the basis of this study, as patients were not randomized and betweengroup differences may be due to selection bias. Moreover, the dose and duration of treatment were left to the discretion of the investigators. Patient assessments were performed in local laboratories, which means that certain baseline characteristics, for example, $I L 28 B$ genotype, were not known for all patients, and that the sensitivity of assays used to determine SVR varied between sites.

In conclusion, the overall efficacy of first-generation PI-based triple-therapy regimens in this real-world study was similar to that reported in other non-interventional studies and somewhat 
Table 9 Sustained virological response at 12 weeks after the end of treatment in treatment-naïve patients with HCV mono-infection receiving dual therapy by baseline characteristics

\begin{tabular}{|c|c|c|c|c|c|c|c|c|}
\hline \multirow{3}{*}{$\begin{array}{l}\text { Baseline } \\
\text { characteristic }\end{array}$} & \multicolumn{8}{|c|}{ Percentage of patients with SVR12 n/N (\%) } \\
\hline & \multicolumn{2}{|c|}{ Genotype 1} & \multicolumn{2}{|c|}{ Genotype 2} & \multicolumn{2}{|c|}{ Genotype 3} & \multicolumn{2}{|c|}{ Genotype 4} \\
\hline & $\begin{array}{c}\text { PegIFN } \\
\text { alfa-2a/ } \\
\text { RBV }\end{array}$ & $\begin{array}{c}\text { PegIFN } \\
\text { alfa- } \\
2 \mathrm{~b} / \mathrm{RBV}\end{array}$ & $\begin{array}{c}\text { PegIFN } \\
\text { alfa-2a/ } \\
\text { RBV }\end{array}$ & $\begin{array}{c}\text { PegIFN alfa- } \\
\text { 2b/RBV }\end{array}$ & $\begin{array}{c}\text { PegIFN } \\
\text { alfa-2a/ } \\
\text { RBV }\end{array}$ & $\begin{array}{c}\text { PegIFN } \\
\text { alfa-2b/ } \\
\text { RBV }\end{array}$ & $\begin{array}{c}\text { PegIFN } \\
\text { alfa-2a/ } \\
\text { RBV }\end{array}$ & $\begin{array}{c}\text { PegIFN } \\
\text { alfa-2b/ } \\
\text { RBV }\end{array}$ \\
\hline \multicolumn{9}{|l|}{ Fibrosis status } \\
\hline Cirrhosis & $\begin{array}{c}76 / 219 \\
(34.7)\end{array}$ & $\begin{array}{l}20 / 52 \\
(38.5)\end{array}$ & $\begin{array}{l}30 / 47 \\
(63.8)\end{array}$ & $14 / 19(73.7)$ & $\begin{array}{c}77 / 146 \\
(52.7)\end{array}$ & $\begin{array}{c}8 / 20 \\
(40.0)\end{array}$ & $\begin{array}{l}33 / 83 \\
(39.8)\end{array}$ & $\begin{array}{c}4 / 11 \\
(36.4)\end{array}$ \\
\hline No cirrhosis & $\begin{array}{c}325 / 595 \\
(54.6)\end{array}$ & $\begin{array}{l}63 / 146 \\
(43.2)\end{array}$ & $\begin{array}{c}173 / 221 \\
(78.3)\end{array}$ & $51 / 59(86.4)$ & $\begin{array}{c}271 / 382 \\
(70.9)\end{array}$ & $\begin{array}{l}52 / 71 \\
(73.2)\end{array}$ & $\begin{array}{c}136 / 257 \\
(52.9)\end{array}$ & $\begin{array}{l}19 / 34 \\
(55.9)\end{array}$ \\
\hline \multicolumn{9}{|c|}{$\begin{array}{l}\text { IL28B rs12979860 } \\
\text { host genotype }\end{array}$} \\
\hline $\mathrm{CC}$ & $\begin{array}{c}135 / 200 \\
(67.5)\end{array}$ & $\begin{array}{l}22 / 35 \\
(62.9)\end{array}$ & $\begin{array}{l}64 / 75 \\
(85.3)\end{array}$ & $16 / 19(84.2)$ & $\begin{array}{c}111 / 154 \\
(72.1)\end{array}$ & $\begin{array}{l}10 / 18 \\
(55.6)\end{array}$ & $\begin{array}{l}24 / 34 \\
(70.6)\end{array}$ & $\begin{array}{c}7 / 9 \\
(77.8)\end{array}$ \\
\hline $\mathrm{CT}$ & $\begin{array}{c}145 / 327 \\
(44.3)\end{array}$ & $\begin{array}{l}16 / 52 \\
(30.8)\end{array}$ & $\begin{array}{l}59 / 84 \\
(70.2)\end{array}$ & $23 / 29(79.3)$ & $\begin{array}{c}103 / 158 \\
(65.2)\end{array}$ & $\begin{array}{l}22 / 31 \\
(71.0)\end{array}$ & $\begin{array}{l}15 / 44 \\
(34.1)\end{array}$ & $\begin{array}{c}7 / 14 \\
(50.0)\end{array}$ \\
\hline TT & $\begin{array}{l}35 / 80 \\
(43.8)\end{array}$ & $\begin{array}{c}8 / 21 \\
(38.1)\end{array}$ & $\begin{array}{l}10 / 16 \\
(62.5)\end{array}$ & $6 / 7(85.7)$ & $\begin{array}{l}24 / 37 \\
(64.9)\end{array}$ & $\begin{array}{c}4 / 8 \\
(50.0)\end{array}$ & $\begin{array}{l}10 / 24 \\
(41.7)\end{array}$ & $\begin{array}{c}1 / 3 \\
(33.3)\end{array}$ \\
\hline Unknown & $\begin{array}{c}85 / 207 \\
(41.1)\end{array}$ & $\begin{array}{l}37 / 90 \\
(41.1)\end{array}$ & $\begin{array}{l}70 / 93 \\
(75.3)\end{array}$ & $20 / 23(87.0)$ & $\begin{array}{c}110 / 179 \\
(61.5)\end{array}$ & $\begin{array}{l}24 / 34 \\
(70.6)\end{array}$ & $\begin{array}{c}120 / 238 \\
(50.4)\end{array}$ & $\begin{array}{c}8 / 19 \\
(42.1)\end{array}$ \\
\hline \multicolumn{9}{|c|}{ HCV RNA (IU/mL) } \\
\hline$\leq 400,000$ & $\begin{array}{c}148 / 234 \\
(63.2)\end{array}$ & $\begin{array}{l}28 / 63 \\
(44.4)\end{array}$ & $\begin{array}{l}77 / 99 \\
(77.8)\end{array}$ & $21 / 24(87.5)$ & $\begin{array}{c}124 / 183 \\
(67.8)\end{array}$ & $\begin{array}{l}19 / 34 \\
(55.9)\end{array}$ & $\begin{array}{c}79 / 139 \\
(56.8)\end{array}$ & $\begin{array}{l}11 / 18 \\
(61.1)\end{array}$ \\
\hline$>400,000$ & $\begin{array}{c}253 / 581 \\
(43.5)\end{array}$ & $\begin{array}{l}55 / 135 \\
(40.7)\end{array}$ & $\begin{array}{c}126 / 169 \\
(74.6)\end{array}$ & $44 / 54(81.5)$ & $\begin{array}{c}223 / 342 \\
(65.2)\end{array}$ & $\begin{array}{l}41 / 57 \\
(71.9)\end{array}$ & $\begin{array}{c}90 / 201 \\
(44.8)\end{array}$ & $\begin{array}{l}12 / 27 \\
(44.4)\end{array}$ \\
\hline
\end{tabular}

SVR12 = percentage of patients with response at least 12 weeks after completion of the treatment period, i.e., HCV RNA $<50 \mathrm{IU} / \mathrm{mL}$ at least 70 days after day of last dose. Response: HCV RNA $<50 \mathrm{IU} / \mathrm{mL}$

HCV, hepatitis C virus; PegIFN, peginterferon; RBV, ribavirin; SVR, sustained virological response

lower than that reported in registration trials, whilst the safety profile of triple therapy was broadly comparable to that in phase III clinical trials. Efficacy and safety with dual-therapy regimens were comparable to previous real-world studies.

\section{Acknowledgments}

The authors thank Fernando Tatsch for his contributions to the initiation and setup of this study while in the employment of F. Hoffmann-La Roche Ltd, Basel, Switzerland.

The authors thank the PegBase Study Group investigators: Belgium: Chantal De Galocsy, Collins Assene, Boris Bastens, Stefan Bourgeois, Christian Brixko, Mike Cool, Jochen Decaestecker, Jean Delwaide, Matthias Dietze, Yves Horsmans, Filip Janssens, Philippe Langlet, Luc Lasser, Véronique Lefebvre, Peter Michielsen, Christophe Moreno, Jean-Pierre Mulkay, Frederik Nevens, Sergio Negrin Dastis, Etienne Wain, Marc Van Hoof, Christophe George, Frédéric Flamme, Christophe Van Steenkiste, Dirk Sprengers; Egypt: Imam Waked, Asem Elfert, Ahmed Monis, Moataz Serri, Ahmed Zeid, Ibrahim Mostafa, Noman Elgarem, Hesham El Khayat, Sherif Abd El Fatah, Ashraf
Omar, Ali Gaballah, Ehab Elshimi, Saleh Mahmoud; Estonia: Riina Salupere, Nele Rasmann, Krista Jaago, Jelena Šmidt, Benno Margus; France: Jean-Pierre Arpurt, Solange Bresson Hadni, Jean-François Cadranel, Xavier Causse, Thong Dao, Victor De Ledinghen, Christophe Renou, Francois Habersetzer, Christophe Hezode, Nathalie Talbodec, Dominique Larrey, Joseph Moussalli, Raluca Pais, Bruno Roche, Dominique Thabut Damais, Regine Truchi, Firouze Bani Sadr, Dominique Batisse, Thierry Constant, Patrick Delasalle, Florence Bouchet Laneuw, Marie Ecochard, Sophie Hillaire, Denis Ouzan, Yves Benhamou, Ghassan Riachi, Faouzi Saliba, Laurent Alric, Serge Bellon, Brigitte Bernard Chabert, Nathalie Boyer Darrigrand, Dominique Capron Chivrac, Nicolas Carbonell, Laetitia Heymann Fartoux, Bertrand Hanslik, Eric Duc Nguyen Khac, Yannick Bacq, Vincent Di Martino, Gilles Pelletier, Helene Donnadieu Rigole, Veronique Loustaud Ratti, Arnaud Pauwels, Philippe Sogni, Pierre Blanc, Corinne Castelnau Marchand, Louis D’Alteroche, Michel Doffoel, Jean Charles Duclos Vallee, Gilles Macaigne, Philippe Mathurin, Valerie Oules Guieu, Pierre Attali, Alina Pascale, Andre Jean Remy, Marc Antoine Valantin, Jean-Pierre Bronowicki, Magali Picon, Stanislas Pol, Ramuntcho Arotcarena, Cathia Joseph Reinette, Philippe Renard, Didier Ribard, Isabelle Rosa, Didier Samuel, Christophe Locher, 
Table 10 Safety outcomes in treatment-naïve and previously treated patients with HCV mono-infection (any genotype) receiving dual therapy

\begin{tabular}{|c|c|c|}
\hline Adverse event & $\begin{array}{c}\text { PegIFN } \\
\text { alfa-2a/RBV } \\
(\mathrm{N}=2312)\end{array}$ & $\begin{array}{c}\text { PegIFN } \\
\text { alfa-2b/RBV } \\
(\mathrm{N}=496)\end{array}$ \\
\hline Patients with $\geq 1$ AE, n (\%) & $1393(60.3)$ & $326(65.7)$ \\
\hline Patients with $\geq 1$ SAE, $n$ (\%) & $148(6.4)$ & $33(6.7)$ \\
\hline Deaths $^{\dagger}, \mathrm{n}(\%)$ & $6(0.3)$ & $3(0.6)$ \\
\hline \multicolumn{3}{|l|}{$\begin{array}{l}\text { Incidence of individual } \mathrm{AEs}^{*} \text {, } \\
\mathrm{n}(\%)\end{array}$} \\
\hline Anemia & $456(19.7)$ & $142(28.6)$ \\
\hline Neutropenia & $270(11.7)$ & $65(13.1)$ \\
\hline Asthenia & $237(10.3)$ & $72(14.5)$ \\
\hline Fatigue & $244(10.6)$ & $53(10.7)$ \\
\hline Pruritus & $160(6.9)$ & $55(11.1)$ \\
\hline $\begin{array}{l}\text { Peginterferon dose } \\
\text { modification } \neq, \mathrm{n}(\%)\end{array}$ & $243(10.5)$ & $65(13.1)$ \\
\hline Neutropenia & $138(6.0)$ & $22(4.4)$ \\
\hline Thrombocytopenia & $46(2.0)$ & $9(1.8)$ \\
\hline Anemia & $14(0.6)$ & $7(1.4)$ \\
\hline Other & $71(3.1)$ & $34(6.9)$ \\
\hline $\begin{array}{l}\text { Ribavirin dose modification }{ }^{\ddagger} \text {, } \\
\mathrm{n}(\%)\end{array}$ & $384(16.6)$ & $120(24.2)$ \\
\hline Anemia & $315(13.6)$ & $101(20.4)$ \\
\hline Other & $95(4.1)$ & $22(4.4)$ \\
\hline \multicolumn{3}{|l|}{$\begin{array}{l}\text { Laboratory abnormalities, } \\
\text { lowest values after BL, n (\%) }\end{array}$} \\
\hline Hemoglobin $<100 \mathrm{~g} / \mathrm{L}$ & $417(18.4)$ & $100(20.7)$ \\
\hline Platelets $<100 \times 10^{9} / \mathrm{L}$ & $716(31.6)$ & $110(22.8)$ \\
\hline Neutrophils $<2.0 \times 10^{9} / \mathrm{L}$ & $1914(86.1)$ & $420(87.9)$ \\
\hline
\end{tabular}

${ }^{*}$ Incidence $\geq 10 \%$ in one or both treatment groups, ${ }^{+}$Total number of deaths regardless of relation to treatment in the opinion of the investigator, ${ }^{*}$ Because of adverse event or laboratory abnormality

$A E$, adverse event; $B L$, baseline; HCV, hepatitis C virus; PegIFN, peginterferon; $R B V$, ribavirin; $S A E$, serious adverse event

Veronique Sitruk, Louis Bettan, Jean-Pierre Crumbach, Sylva Lahoud Doumet, Abdelkrim Medini, Marie Pierre Ripault, Stephanie Dominguez, Dominique Guyader, Julie Chas, Michel Antoni, Philippe Chassagne, Thierry Fontanges, Benedicte Lambare, Sylvain Beorchia, Nathalie Giuily Guigui, David Parlier, Jean Jacques Laurichesse, Dominique Salmon Ceron, Gaelle Billet, Michele Pouteau; Germany: Tobias Goeser, Guido Gerken, Jörg Schlaak, Christian Trautwein, Steffen Zopf, Klaus Schmidt, Stefan Mauss, Christoph Berg, Christoph Antoni, Christiane Cordes, Michael-Ruppert Kraus, Luca Stein, Stefan Strahl, Manfred Kuhn, Kerstin Stein, Micha Loebermann; Greece: Dimitrios Dimitroulopoulos, George Papatheodoridis, Ioannis Goulis, Konstantinos Mimidis, Epameinondas Tsianos, Georgios Dalekos, Vassiliki Nikolopoulou, Ioannis Ketikoglou, Maria Raptopoulou-Gigi, Ioannis Elefsiniotis; Hungary:
Erzsébet Makkai, Pál Ribiczey, Marta Varga, Judit Enyedi, Gabriella Lengyel, Gyula Tolvaj, János Schuller, Krisztina Nemesi, Ferenc Szalay, Istvan Tornai, Tivadar Banyai, Bela Hunyady, Zsuzsanna Váczi, Miklos Lesch, Aron Vincze, Arpád Patai, Zsófia Ozsvár, Judit Gervain, Fejér Megyei, Anna Tusnadi, László Szentgyörgyi, Lilla Lakner, Ferenc Schneider, Elemér Nemesánszky, Istvan Varkonyi, Gábor Horváth; Ireland: Suzanne Norris, Frank Murray, John Hegarty; Italy: Arnaldo Andreoli, Adolfo Francesco Attili, Danilo Tacconi, Cosimo Colletta, Carmine Coppola, Raffaele Cozzolongo, Piero Almasio, Luigi Demelia, Pietro Di Cicco, Giuseppe Foti, Roberto Ganga, Anna Giannelli, Massimo Memoli, Lydia Giannitrapani, Antonio Picciotto, Antonello Pietrangelo, Alessandra Mangia, Tiziana Quirino, Giovanni Raimondo, Giuliano Rizzardini, Elke Erne, Giorgio Soardo, Massimo Zuin, Gabriella Verucchi, Silvia Fargion, Ludovico Tallarico, Massimo Puoti, Sergio Babudieri, Luigi Elio Adinolfi, Spartaco Sani, Maurizio Russello, Giovan Giuseppe Di Costanzo, Alfredo Di Leo, Francesco Di Lorenzo, Paolo Forleo, Giuseppe D’Adamo, Davide Drenaggi; Ubaldo Visco Comandini, Maria Grazia Rumi, Antonio Gasbarrini, Bruno Cacopardo, Gianfranco Delle Fave, Fabio Fornari, Alessandro Grasso, Vincenzo Ostilio Palmieri, Sergio Peyre, Jacopo Vecchiet, Alessandra Orlandini, Aldo Marrone, Massimo Andreoni, Nicola Passariello, Salvatore D’Angelo, Giuseppe Mazzella; Kuwait: Haifa Askar; Lebanon: Ala Sharara, Fouad Zaarour, Zaher Houmani, Iyad Issa, Ahmad Dohaibi, Cesar Yaghi, Antoine Abou Rached; Former Yugoslav Republic of Macedonia: Viktorija Chaloska-Ivanova, Magdalena Gaseva; Morocco: Abdeaziz Ziane, Driss Jamil, Essaid Abdellah, Majida Zahraoui, Abdelmajid Sebti, Med Jalil Benkirane, Khadija Krati, Nourdin Aqodad, Med Kamal Benhayoun, Salwa Nadir, Mhammed Nya, Nawal Kabbaj, Rajaa Afifi, Hana Benbrahim, Mostafa Benazzouz, Laila Amrani; Oman: Khalid Al Naamani; Pakistan: Zaigham Abbas, Asad Chaudhry, Anwaar Khan, Altaf Alam, Asghar Auranzeb Durrani, Furqaan Ahmed, GhiasUn Nabi Tayyab, Zahid Hashmi; Portugal: Rita Ornelas, Jorge Velez, Crisitina Valente, Paula Ferreira, Tiago Bana, Alexandra Martins, Carlos Monteverde, Maria João Aleixo, Isabel Pedroto, Kamal Mansinho; Qatar: Mouneera Al Mohandi; Romania: Manuela Curescu, Liana Gheorghe, Anca Trifan, Eugen Dumitru, Cristina Cijevschi, Mircea Grigorescu; Saudi Arabia: Khalid Al Kahtani, Mohammed Kareem Sharif, Adnan Al Zanbagi, Khalid Bzeizi; Serbia: Milotka Fabri, Petar Svorcan; Sweden: Lars Wesslén, Anders Rubenson, Anders Lannergard; Switzerland: Andreas Cerny, David Semela; Syrian Arab Republic: Nabil Antaki; Taiwan: Jia-Horng Kao, Wan-Long Chuang, I-Shyan Sheen, Chi-Jen C. Chu, Cheng-Yuan Peng, Tsung-Hui Hu; Turkey: Abdulkadir Dokmeci, Meral Akdogan, Yusuf Onlen, Orhan Sezgin, Iftihar Koksal, Sener Barut, Resat Ozaras, Rahmet Guner, Hayat Kumbasar, Ulus Akarca, Sebnem Gursoy, Mehmet Demir; United Arab Emirates: Talaat Deyab, Thomas Cherukara, Elhassan Sidahmed Elhassan Yousif; United Kingdom: Graham Foster, Stephen Ryder, Alison Uriel, John Dillon, Ashley Brown, George Abouda

Support for third-party writing assistance for this manuscript, furnished by Blair Jarvis MSc, ELS, of Health Interactions, was provided by F. Hoffmann-La Roche Ltd., Basel, Switzerland. 


\section{Summary Box}

\section{What is already known:}

- When added to peginterferon alfa/ribavirin, the first direct-acting antiviral agents for chronic hepatitis $\mathrm{C}$ (boceprevir and telaprevir) increased sustained virological response (SVR) rates in genotype 1 patients and reduced the duration of treatment required to maximize SVR rates

- First-generation protease inhibitor (PI)-based triple therapy is associated with a higher adverse event burden than dual peginterferon alfa/ribavirin therapy

- SVR rates are higher in treatment-naïve patients than in previously treated patients

- Previous cohort studies of first-generation PI-based triple therapy reported lower SVR rates than those achieved in registration studies

\section{What the new findings are:}

- The results of this large real-world observational trial of boceprevir- and telaprevir-based triple therapy in a heterogeneous "real-world" population are consistent with previous, smaller cohort studies that have reported somewhat lower SVR rates than those obtained in phase III registration studies

- The tolerability profile of boceprevir- and telaprevir-based triple therapy in a real-world setting is similar to that reported in registration studies

- When triple therapy is prescribed in accordance with local standards of practice, as recommended in this trial, and the approved label, as in the present study, the incidence of hepatic decompensation and death is low

\section{References}

1. World Health Organization (WHO). Guidelines for the screening, care and treatment of persons with hepatitis C infection. April 2014. Available at: http://www.who.int/hiv/pub/hepatitis/hepatitis-cguidelines/en/. Last accessed: 3 March 2017.

2. Messina JP, Humphreys I, Flaxman A, et al. Global distribution and prevalence of hepatitis C virus genotypes. Hepatology 2015;61:77-87.

3. Thein HH, Yi Q, Dore GJ, Krahn MD. Estimation of stage-specific fibrosis progression rates in chronic hepatitis $\mathrm{C}$ virus infection: a meta-analysis and meta-regression. Hepatology 2008;48:418-431.

4. El-Serag HB, Rudolph KL. Hepatocellular carcinoma: epidemiology and molecular carcinogenesis. Gastroenterology 2007;132:2557-2576.

5. Cooke GS, Lemoine M, Thursz M, et al. Viral hepatitis and the Global Burden of Disease: a need to regroup. J Viral Hepat 2013;20:600-601.

6. Jacobson IM, McHutchison JG, Dusheiko G, et al; ADVANCE Study Team. Telaprevir for previously untreated chronic hepatitis $C$ virus infection. N Engl J Med 2011;364:2405-2416.

7. Poordad F, McCone J Jr, Bacon BR, et al; SPRINT-2 Investigators. Boceprevir for untreated chronic HCV genotype 1 infection. $N$ Engl
J Med 2011;364:1195-1206.

8. Bacon BR, Gordon SC, Lawitz E, et al; HCV RESPOND-2 Investigators. Boceprevir for previously treated chronic HCV genotype 1 infection. N Engl J Med 2011;364:1207-1217.

9. Zeuzem S, Andreone P, Pol S, et al; REALIZE Study Team. Telaprevir for retreatment of HCV infection. N Engl J Med 2011;364:2417-2428.

10. American Association for the Study of Liver Diseases (AASLD) \& Infectious Diseases Society of America (IDSA). HCV Guidance: Recommendations for testing, managing, and treating hepatitis C. Updated 2016. Available at: http://www.hcvguidelines.org/fullreport. Accessed June 2016.

11. European Association for Study of Liver. EASL Recommendations on Treatment of Hepatitis C 2015. J Hepatol 2015;63:199-236.

12. Omata M, Kanda T, Wei L, et al. APASL consensus statements and recommendation on treatment of hepatitis C. Hepatol Int 2016;10:702-726.

13. European Association for Study of Liver. EASL Clinical Practice Guidelines: management of hepatitis C virus infection. J Hepatol 2014;60:392-420.

14. Sherman KE, Flamm SL, Afdhal NH, et al; ILLUMINATE Study Team. Response-guided telaprevir combination treatment for hepatitis C virus infection. N Engl J Med 2011;365:1014-1024.

15. Shiffman ML, Di Bisceglie AM, Lindsay KL, et al; Hepatitis C Antiviral Long-Term Treatment Against Cirrhosis Trial Group. Peginterferon alfa-2a and ribavirin in patients with chronic hepatitis C who have failed prior treatment. Gastroenterology 2004;126:10151023; discussion 947 .

16. Jacobson IM, Gonzalez SA, Ahmed F, et al. A randomized trial of pegylated interferon alpha- $2 \mathrm{~b}$ plus ribavirin in the retreatment of chronic hepatitis C. Am J Gastroenterol 2005;100:2453-2462.

17. Poynard T, Colombo M, Bruix J, et al; Epic Study Group. Peginterferon alfa- $2 b$ and ribavirin: effective in patients with hepatitis $C$ who failed interferon alfa/ribavirin therapy. Gastroenterology 2009;136:16181628.e2.

18. Poordad F, Bronowicki JP, Gordon SC, et al; SPRINT-2 and RESPOND-2 Investigators. Factors that predict response of patients with hepatitis $\mathrm{C}$ virus infection to boceprevir. Gastroenterology 2012;143:608-618.e1-e5.

19. Hézode C, Fontaine H, Dorival C, et al; CUPIC Study Group. Triple therapy in treatment-experienced patients with HCV-cirrhosis in a multicentre cohort of the French Early Access Programme (ANRS CO20-CUPIC) - NCT01514890. J Hepatol 2013;59:434-441.

20. Hézode C, Fontaine H, Dorival C, et al; CUPIC Study Group. Effectiveness of telaprevir or boceprevir in treatment-experienced patients with HCV genotype 1 infection and cirrhosis. Gastroenterology 2014;147:132-142.e4.

21. Price JC, Murphy RC, Shvachko VA, Pauly MP, Manos MM. Effectiveness of telaprevir and boceprevir triple therapy for patients with hepatitis $C$ virus infection in a large integrated care setting. Dig Dis Sci 2014;59:3043-3052.

22. Gordon SC, Muir AJ, Lim JK, et al; HCV-TARGET study group. Safety profile of boceprevir and telaprevir in chronic hepatitis C: real world experience from HCV-TARGET. J Hepatol 2015;62:286-293.

23. Mauss S, Böker K, Buggisch P, et al. Real-life experience with first generation HCV protease inhibitor therapy in Germany: The prospective, non-interventional PAN cohort. Z Gastroenterol 2015;53:644-654.

24. Manns MP, McHutchison JG, Gordon SC, et al. Peginterferon alfa-2b plus ribavirin compared with interferon alfa- $2 \mathrm{~b}$ plus ribavirin for initial treatment of chronic hepatitis C: a randomised trial. Lancet 2001;358:958-965.

25. Fried MW, Shiffman ML, Reddy KR, et al. Peginterferon alfa-2a plus ribavirin for chronic hepatitis C virus infection. $N$ Engl J Med 2002;347:975-982.

26. Hadziyannis SJ, Sette H Jr, Morgan TR, et al; PEGASYS International Study Group. Peginterferon-alpha2a and ribavirin combination therapy in chronic hepatitis C: a randomized study of treatment duration and ribavirin dose. Ann Intern Med 2004;140:346-355.

27. Marcellin P, Cheinquer H, Curescu M, et al. High sustained virologic response rates in rapid virologic response patients in the large realworld PROPHESYS cohort confirm results from randomized clinical trials. Hepatology 2012;56:2039-2050. 


\section{Supplemental Tables}

Supplemental Table 1 Definition of the core population

Adult male or female patients with chronic hepatitis C (CHC), hepatitis C virus (HCV) mono-infection with known HCV genotype and known pre-treatment status (naïve or previously treated) who gave informed consent and received one of the following treatment regimens exclusively:

- Peginterferon alfa-2a + ribavirin

- Peginterferon alfa- $2 \mathrm{~b}+$ ribavirin

- Boceprevir + peginterferon alfa-2a + ribavirin

- Boceprevir + peginterferon alfa- $2 b+$ ribavirin

- Telaprevir + peginterferon alfa-2a + ribavirin

- Telaprevir + peginterferon alfa- $2 b+$ ribavirin

Excluded patients:

CHC was not confirmed (i.e., no positive baseline HCV RNA record, or acute hepatitis C)

Contraindications for any drug in the assigned regimen as per prescribing information (dual peginterferon plus ribavirin therapy or the directacting antiviral-containing regimen)

End-stage renal disease

Major organ transplantation

Hepatitis B virus coinfection

HIV coinfection or unknown HIV infection status

Infection with a non-1 HCV genotype and receipt of boceprevir- or telaprevir-based triple combination therapy

Treatment with a combination other than the six described above

Missing final confirmation of the data by the principal investigator

Supplemental Table 2 Reasons for exclusion of treated patients from the core population (safety population)

\begin{tabular}{lc}
\hline $\begin{array}{l}\text { Reason for exclusion (more than one reason may } \\
\text { apply) }\end{array}$ & $\mathrm{N}=252$ \\
\hline HIV co-infection & 131 \\
\hline $\begin{array}{l}\text { No treatment or treatment other than Peg-IFN } \\
\text { alfa-2a + RBV or Peg-IFN alfa-2b + RBV or one } \\
\text { of these two regimens plus either boceprevir or } \\
\text { telaprevir }\end{array}$ & 23 \\
$\begin{array}{l}\text { Not HCV RNA-positive at baseline } \\
\begin{array}{l}\text { Triple therapy administered to non-genotype 1 } \\
\text { patient }\end{array}\end{array}$ & 14 \\
$\begin{array}{l}\text { No final confirmation by principal investigator } \\
\text { Non-adult CHC patient receiving dual or triple } \\
\text { therapy }\end{array}$ & 29 \\
\hline HCV genotype missing & 1 \\
\hline $\begin{array}{l}\text { Contraindication to one or more therapies in the } \\
\text { assigned regimen }\end{array}$ & 9 \\
\hline $\begin{array}{l}\text { HBV co-infection } \\
\text { Response at baseline }\end{array}$ & 10 \\
\hline End-stage renal disease & 6 \\
\hline Major organ transplantation & 4 \\
\hline
\end{tabular}

